\title{
Diagnostic accuracy of history taking, physical examination and imaging for phalangeal, metacarpal and carpal fractures: a systematic review update
}

Patrick Krastman $^{1 *}$ (D, Nina M. Mathijssen², Sita M. A. Bierma-Zeinstra ${ }^{3,4}$, Gerald Kraan² and Jos Runhaar ${ }^{1}$

\begin{abstract}
Background: The standard diagnostic work-up for hand and wrist fractures consists of history taking, physical examination and imaging if needed, but the supporting evidence for this work-up is limited. The purpose of this study was to systematically examine the diagnostic accuracy of tests for hand and wrist fractures.

Methods: A systematic search for relevant studies was performed. Methodological quality was assessed and sensitivity (Se), specificity (Sp), accuracy, positive predictive value (PPV) and negative predictive value (NPV) were extracted from the eligible studies.

Results: Of the 35 eligible studies, two described the diagnostic accuracy of history taking for hand and wrist fractures. Physical examination with or without radiological examination for diagnosing scaphoid fractures (five studies) showed Se, Sp, accuracy, PPV and NPV ranging from 15 to 100\%, 13-98\%, 55-73\%, 14-73\% and 75-100\%, respectively. Physical examination with radiological examination for diagnosing other carpal bone fractures (one study) showed a Se of 100\%, with the exception of the triquetrum (75\%). Physical examination for diagnosing phalangeal and metacarpal fractures (one study) showed Se, Sp, accuracy, PPV and NPV ranging from 26 to 55\%, 13-89\%, 45-76\%, 41-77\% and 63-75\%, respectively.

Imaging modalities of scaphoid fractures showed predominantly low values for PPV and the highest values for Sp and NPV (24 studies). Magnetic Resonance Imaging (MRI), Computed Tomography (CT), Ultrasonography (US) and Bone Scintigraphy (BS) were comparable in diagnostic accuracy for diagnosing a scaphoid fracture, with an accuracy ranging from 85 to 100\%, 79-100\%, 49-100\% and 86-97\%, respectively. Imaging for metacarpal and finger fractures showed Se, Sp, accuracy, PPV and NPV ranging from 73 to 100\%, 78-100\%, 70-100\%, 79-100\% and 70-100\%, respectively.

Conclusions: Only two studies were found on the diagnostic accuracy of history taking for hand and wrist fractures in the current review. Physical examination was of moderate use for diagnosing a scaphoid fracture and of limited use for diagnosing phalangeal, metacarpal and remaining carpal fractures. MRI, CT and BS were found to be moderately accurate for the definitive diagnosis of clinically suspected carpal fractures.
\end{abstract}

Keywords: Diagnostic tests, Finger, Fracture, Hand, Wrist

\footnotetext{
* Correspondence: wetenschap@dezorghoek.nl

${ }^{1}$ Department of General Practice, Erasmus MC University Medical Center

Rotterdam, Room NA1911 PO Box 2040, 3000, CA, Rotterdam, the

Netherlands

Full list of author information is available at the end of the article
}

(c) The Author(s). 2020 Open Access This article is distributed under the terms of the Creative Commons Attribution 4.0 International License (http://creativecommons.org/licenses/by/4.0/), which permits unrestricted use, distribution, and reproduction in any medium, provided you give appropriate credit to the original author(s) and the source, provide a link to the Creative Commons license, and indicate if changes were made. The Creative Commons Public Domain Dedication waiver (http://creativecommons.org/publicdomain/zero/1.0/) applies to the data made available in this article, unless otherwise stated. 


\section{Background}

Hand and wrist injuries are among the most common traumatic presentations to the emergency department $[1,2]$, and commonly affect young people of working age [3, 4]. Scaphoid fractures are the most frequently injured carpal bones, accounting for $61-90 \%$ of fractures [4-6]. The diagnosis of a scaphoid fracture may however be difficult to establish on a conventional radiograph [7, 8]. Previous research has shown that $10-35 \%$ of scaphoid fractures are missed on primary radiographs [4, 9-12]. Metacarpal fractures are detected in $30-40 \%$ of all hand fractures in all emergency department admissions $[4,9,10]$.

Hand and wrist injuries represent a considerable economic burden, with high health-care and productivity costs [13]. The total costs have been estimated at US $\$ 410$ million per year, with US $\$ 307$ million in productivity costs [14].

If not treated properly, patients with hand and wrist injuries may experience lifelong pain and lose their job, which also has major effects on their quality of life [15]. Accurate diagnosis and early treatment of hand and wrist fractures are important because missed diagnosis and delayed initiation of therapy increase the risk of complications and subsequent functional impairment [16-22].

In recent decades, research has predominantly focused on imaging modalities for the diagnosis of wrist fractures. However, the standard diagnostic work-up for wrist complaints that are suspected fractures should also include detailed patient history taking, a conscientious physical examination and, only if needed, imaging [23]. It has been shown that different provocative tests are somewhat useful for diagnosing wrist fractures [24-27], but there is no consensus on imaging protocols due to limited evidence regarding the diagnostic performance of these advanced imaging techniques [28]. Therefore, diagnosing wrist pathologies remain complex and challenging and there is increasing demand for evidence for accurate diagnostic tools [29].

Diagnostic studies performed in hospital care cannot automatically be translated into guidelines for noninstitutionalized general practitioner care [30]. The clinical utility of diagnostic tests for hand and wrist fractures is hindered by the low prevalence of true fractures, approximately $7 \%$ on average [31].

Currently, there are several systematic reviews available on the diagnostic accuracy of tests for the diagnosis of hand and wrist fractures, as presented in Table 1 [32-39]. Of these, only the review by Carpenter et al. used 'history' as a keyword in their search terms, but they could not find studies assessing the diagnostic accuracy of history for scaphoid fractures [32]. All the available systematic reviews only examined diagnostic tests for scaphoid fractures [32-39], while in practice it is often not quite clear during the diagnostic process which hand or wrist anatomical structure or tissue (soft tissue or bone) is affected. Moreover, these reviews focused predominantly on imaging as a diagnostic tool, while in clinical practice a diagnosis is mainly made on history taking and physical examination.

Therefore, the purpose of this literature review is to provide an up-to-date systematic overview of the diagnostic accuracy of history taking, physical examination and imaging for phalangeal, metacarpal and carpal fractures and to distinguishing between studies in hospital and non-institutionalized general practitioner care settings, as test properties may differ between settings. Compared to previously published reviews, in this systematic review we also included studies that examined history taking and physical examination for phalangeal, metacarpal or carpal fractures.

\section{Methods \\ Data sources and searches}

A review protocol was drafted, but central registration was not completed. The Preferred Reporting Items for Systematic Reviews and Meta-Analyses (PRISMA) Statement was used to guide the conduct and reporting of the study [40]. A Biomedical Information specialist (Wichor M. Bramer) performed a search for studies in Medline, Embase, Cochrane Library, Web of Science, Google Scholar ProQuest and Cinahl from 2000 up to 6 February 2019. This starting point was used since multiple reviews are available that already cover the period up to the year 2000 (Table 1). Search terms included phalangeal, metacarpal and carpal injuries, anamnestic assessment, provocative test(s), diagnostic test(s) and imaging tests. The full electronic search strategy for the Embase database is presented in Table 2 (the others are available upon request).

\section{Study selection}

Studies describing diagnostic accuracy of history taking, physical examination or imaging in adult patients (age $\geq$ 16 years) with phalangeal, metacarpal and/or carpal fractures were included. No language restriction was applied. Case reports, reviews and conference proceedings were excluded. Distal radius and ulna injuries were also excluded, as they can be diagnosed accurately with plane $\mathrm{X}$-ray or computer tomography imaging.

Two reviewers (PK, YA) read all titles and abstracts independently. Articles that could not be excluded on the basis of the title and/or abstract were retrieved in full text and were read and checked for inclusion by the two reviewers independently. If there was no agreement, a third reviewer (JR) made the final decision. In addition, the reference lists of all included studies were reviewed to check for additional relevant studies. 
Table 1 Characteristics of the Currently Available Systematic Reviews on the Diagnostic Accuracy of Tests

\begin{tabular}{|c|c|c|c|c|c|c|c|c|}
\hline Author(s) & $\begin{array}{l}\text { Population in eligible } \\
\text { studies as described } \\
\text { by the review authors }\end{array}$ & Fracture & $\begin{array}{l}\text { Number } \\
\text { of studies } \\
\text { included }\end{array}$ & $\begin{array}{l}\text { Diagnostic } \\
\text { test }\end{array}$ & $\begin{array}{l}\text { Pooled Se } \\
(95 \% \mathrm{Cl})\end{array}$ & $\begin{array}{l}\text { Pooled Sp } \\
(95 \% \text { CI) }\end{array}$ & Positive LR & Conclusion \\
\hline \multicolumn{9}{|l|}{ HISTORY TAKING } \\
\hline $\begin{array}{l}\text { Carpenter } \\
\text { (2014) [32] }\end{array}$ & $\begin{array}{l}\text { Emergency } \\
\text { Department. }\end{array}$ & Scaphoid & 0 & & & & & $\begin{array}{l}\text { History examination alone is } \\
\text { inadequate to rule in or rule } \\
\text { out scaphoid fracture. }\end{array}$ \\
\hline \multicolumn{9}{|c|}{ PHYSICAL EXAMINATION } \\
\hline \multirow{7}{*}{$\begin{array}{l}\text { Carpenter } \\
\text { (2014) [32] }\end{array}$} & \multirow{7}{*}{$\begin{array}{l}\text { Emergency } \\
\text { Department. }\end{array}$} & \multirow[t]{7}{*}{ Scaphoid } & 6 & ASB tenderness & $0.96(0.92-0.98)$ & $0.39(0.36-0.43)$ & & \multirow{7}{*}{$\begin{array}{l}\text { Except for the absence of } \\
\text { snuffbox tenderness, which } \\
\text { can significantly reduce the } \\
\text { probability of scaphoid } \\
\text { fracture, physical examination } \\
\text { alone is inadequate to rule in } \\
\text { or rule out scaphoid fracture. }\end{array}$} \\
\hline & & & 6 & LTC & $0.82(0.77-0.87)$ & $0.58(0.54-0.62)$ & & \\
\hline & & & 7 & $\begin{array}{l}\text { Ultrasound } \\
\text { fibration pain }\end{array}$ & $0.67(0.59-0.75)$ & $0.57(0.51-0.62)$ & & \\
\hline & & & 3 & Clamp sign & $0.73(0.67-0.78)$ & $0.92(0.89-0.95)$ & & \\
\hline & & & 3 & $\begin{array}{l}\text { Painfull ulnar } \\
\text { deviation }\end{array}$ & $0.77(0.68-0.83)$ & $0.42(0.34-0.49)$ & & \\
\hline & & & 3 & $\mathrm{STT}$ & $0.92(0.86-0.96)$ & $0.47(0.43-0.52)$ & & \\
\hline & & & 2 & $\begin{array}{l}\text { Resisted } \\
\text { supination } \\
\text { pain }\end{array}$ & $0.94(0.85-0.98)$ & $0.74(0.63-0.84)$ & & \\
\hline \multirow{3}{*}{$\begin{array}{l}\text { Burrows } \\
\text { (2014) [33] }\end{array}$} & \multirow[t]{3}{*}{ Not specified } & \multirow[t]{3}{*}{ Scaphoid } & 5 & ASB tenderness & & & $1.52(1.12-2.06)$ & \multirow{3}{*}{$\begin{array}{l}\text { Three clinical tests with } \\
\text { statistically significant } \\
\text { diagnostic validity were } \\
\text { identified. In isolation, the } \\
\text { clinical significance of each is } \\
\text { questionable. }\end{array}$} \\
\hline & & & 7 & $\begin{array}{l}\text { Scaphoid } \\
\text { compression } \\
\text { test }\end{array}$ & & & $2.37(1.27-4.41)$ & \\
\hline & & & 3 & $\mathrm{STT}$ & & & $1.67(1.33-2.09)$ & \\
\hline \multirow{5}{*}{$\begin{array}{l}\text { Mallee } \\
\text { (2015) [34] }\end{array}$} & \multirow{5}{*}{$\begin{array}{l}\text { Patients presenting } \\
\text { to the emergency } \\
\text { department or } \\
\text { outpatient clinic }\end{array}$} & \multirow[t]{5}{*}{ Scaphoid } & 8 & ASB tenderness & $0.87-1.00^{a}$ & $0.03-0.98^{b}$ & & \multirow{5}{*}{$\begin{array}{l}\text { Anatomical snuff box } \\
\text { tenderness was the most } \\
\text { sensitive clinical test. The low } \\
\text { specificity of the clinical tests } \\
\text { may result in a considerable } \\
\text { number of over-treated } \\
\text { patients. Combining tests } \\
\text { improved the post-test } \\
\text { fracture probability. }\end{array}$} \\
\hline & & & 8 & LTC & $0.48-1.00^{\mathrm{a}}$ & $0.22-0.97^{b}$ & & \\
\hline & & & 4 & $\mathrm{STT}$ & $0.82-1.00^{\mathrm{a}}$ & $0.17-0.57^{b}$ & & \\
\hline & & & 4 & $\begin{array}{l}\text { Painfull ulnar } \\
\text { deviation }\end{array}$ & $0.67-1.00^{\mathrm{a}}$ & $0.17-0.60^{b}$ & & \\
\hline & & & 4 & ASB swelling & $0.67-0.77^{\mathrm{a}}$ & $0.37-0.72^{b}$ & & \\
\hline \multicolumn{9}{|l|}{ IMAGING } \\
\hline \multirow{5}{*}{$\begin{array}{l}\text { Carpenter } \\
\text { (2014) [32] }\end{array}$} & \multirow{5}{*}{$\begin{array}{l}\text { Emergency } \\
\text { Department. }\end{array}$} & \multirow[t]{5}{*}{ Scaphoid } & 5 & X-ray fat pad & $0.82(0.76-0.86)$ & $0.72(0.68-0.75)$ & & \multirow{5}{*}{$\begin{array}{l}\text { MRI is the most accurate } \\
\text { imaging test to diagnose } \\
\text { scaphoid fractures in ED } \\
\text { patients with no evidence of } \\
\text { fracture on initial x-rays. If } \\
\text { MRI is unavailable, CT is ad } \\
\text { equate to rule in scaphoid } \\
\text { fractures, but inadequate for } \\
\text { ruling out scaphoid fractures. }\end{array}$} \\
\hline & & & 18 & BS & $0.91(0.87-0.94)$ & $0.86(0.83-0.88)$ & & \\
\hline & & & 6 & US & $0.80(0.67-0.90)$ & $0.87(0.81-0.91)$ & & \\
\hline & & & 8 & CT & $0.83(0.83-0.89)$ & $0.97(0.94-0.98)$ & & \\
\hline & & & 13 & MRI & $0.96(0.92-0.99)$ & $0.98(0.96-0.99)$ & & \\
\hline \multirow[t]{4}{*}{ Yin (2012) [35] } & \multirow[t]{4}{*}{ Not specified } & \multirow[t]{4}{*}{ Scaphoid } & 28 & $\begin{array}{l}\text { Follow-up } \\
\text { radiographs }\end{array}$ & $0.91(0.81-0.98)$ & $1.00(0.99-1.00)$ & & \multirow{4}{*}{$\begin{array}{l}\text { If we acknowledge the lack } \\
\text { of a reference standard for } \\
\text { diagnosing suspected } \\
\text { scaphoid fractures, MRI is the } \\
\text { most accurate test; follow-up } \\
\text { radiographs and CT may be } \\
\text { less sensitive, and bone } \\
\text { scintigraphy less specific. }\end{array}$} \\
\hline & & & 18 & BS & $0.98(0.96-0.99)$ & $0.94(0.91-0.95)$ & & \\
\hline & & & 15 & MRI & $0.98(0.95-0.99)$ & $1.00(0.99-1.00)$ & & \\
\hline & & & 9 & $\subset \mathrm{T}$ & $0.85(0.74-0.94)$ & $1.00(0.98-1.00)$ & & \\
\hline \multirow[t]{3}{*}{ Yin (2010) [36] } & \multirow[t]{3}{*}{ Not specified } & \multirow[t]{3}{*}{ Scaphoid } & 15 & BS & $0.97(0.93-0.99)$ & $0.89(0.83-0.94)$ & & \multirow{3}{*}{$\begin{array}{l}\text { Bone scintigraphy and MRI } \\
\text { have equally high sensitivity } \\
\text { and high diagnostic value for } \\
\text { excluding scaphoid fracture; } \\
\text { however, MRI is more specific } \\
\text { and better for confirming } \\
\text { scaphoid fracture. }\end{array}$} \\
\hline & & & 10 & MRI & $0.96(0.91-0.99)$ & $0.99(0.96-1.00)$ & & \\
\hline & & & 6 & CT & $0.93(0.83-0.98)$ & $0.99(0.96-1.00)$ & & \\
\hline Mallee & People of all ages & Scaphoid & 6 & BS & $0.99(0.69-1.00)$ & $0.86(0.73-0.94)$ & & Bone scintigraphy is \\
\hline & $\begin{array}{l}\text { who presented at } \\
\text { hospital or clinic }\end{array}$ & & 4 & CT & $0.72(0.36-0.92)$ & $0.99(0.71-1.00)$ & & $\begin{array}{l}\text { statistically the best } \\
\text { diagnostic modality to }\end{array}$ \\
\hline & & & 5 & MRI & $0.88(0.64-0.97)$ & $1.00(0.38-1.00)$ & & $\begin{array}{l}\text { establish a definitive } \\
\text { diagnosis in clinically } \\
\text { suspected fractures when } \\
\text { radiographs appear normal. } \\
\text { The number of overtreated } \\
\text { patients is substantially lower }\end{array}$ \\
\hline
\end{tabular}


Table 1 Characteristics of the Currently Available Systematic Reviews on the Diagnostic Accuracy of Tests (Continued)

\begin{tabular}{|c|c|c|c|c|c|c|c|}
\hline Author(s) & $\begin{array}{l}\text { Population in eligible } \\
\text { studies as described } \\
\text { by the review authors }\end{array}$ & Fracture & $\begin{array}{l}\text { Number } \\
\text { of studies } \\
\text { included }\end{array}$ & $\begin{array}{l}\text { Diagnostic } \\
\text { test }\end{array}$ & $\begin{array}{l}\text { Pooled Se } \\
(95 \% \mathrm{Cl})\end{array}$ & $\begin{array}{l}\text { Pooled Sp } \\
(95 \% \text { CI) }\end{array}$ & Conclusion \\
\hline & & & & & & & with $\mathrm{CT}$ and MRI. \\
\hline $\begin{array}{l}\text { Kwee } \\
\text { (2018) [37] }\end{array}$ & Not specified & Scaphoid & 7 & US & $0.86(0.74-0.93)$ & $0.84(0.72-0.91)$ & $\begin{array}{l}\text { Ultrasound can diagnose } \\
\text { radiographically occult } \\
\text { scaphoid fracture with a fairly } \\
\text { high degree of accuracy. }\end{array}$ \\
\hline Ali (2018) [38] & Not specified & Scaphoid & 6 & US & $0.94(0.78-1.00)$ & $0.89(0.78-1.00)$ & $\begin{array}{l}\text { US reveals high sensitivity } \\
\text { and specificity in scaphoid } \\
\text { fracture diagnosis. }\end{array}$ \\
\hline
\end{tabular}

ASB Anatomic snuff-box, LTC Longitudinal (thumb) compression test, STT Scaphoid tubercle tenderness, BS Bone Scintigraphy, US Ultrasound, CT Computed TomographyMRI: Magnetic Resonance Imaging

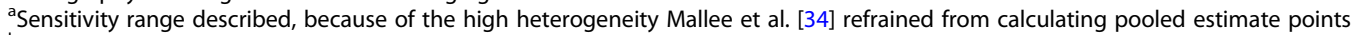

${ }^{\mathrm{b}}$ Specificity Range described, because of the high heterogeneity Mallee et al. [34] refrained from calculating pooled estimate points

\section{Data extraction and methodological quality assessment}

Two reviewers (PK, JR) independently extracted the data. Data were extracted describing the study design, characteristics of the study population, test characteristics, study population setting (hospital care or noninstitutionalized general practitioner care) and diagnostic parameters. Methodological quality was assessed by two independent reviewers (PK, JR), using the Quality Assessment of Diagnostic Accuracy Studies (QUADAS-2) checklist [41]. Disagreements were resolved by discussion.

\section{Heterogeneity}

Key factors in a meta-analysis are the number and the methodological quality of the included studies and the degree of heterogeneity in their estimates of diagnostic accuracy [42]. Heterogeneity in diagnostic test accuracy reviews is expected and the possibilities of performing meta-regression analyses will depend on the number of studies available for a specific index test that provide sufficient information [39]. The data from the included studies were combined when studies showed no limitations according to QUADAS-2 and had no other forms of bias (e.g. incorporation bias).

\section{Data synthesis and analysis}

The following values were extracted, if documented: sensitivity (Se), specificity (Sp), accuracy, positive predictive value (PPV), negative predictive value (NPV) and likelihood ratio (LR). If these diagnostic outcomes were not reported, they were calculated using published data. If an included study presented results from multiple independent observers, the measures of Se, Sp, accuracy, PPV and NPV were averaged over the observers.

\section{Index test}

Diagnostic tools such as history taking, physical examination or imaging were accepted as index tests.

\section{Reference standard}

There is no consensus about the reference test for the diagnosis of a true fracture of the phalangeal, metacarpal or carpal bones [35]. Therefore, in this systematic review clinical outcome (physical examination or additional treatment) and/or various (combined) imaging modalities during follow-up were used as the reference standard for confirming diagnosis of phalangeal, metacarpal or carpal fractures.

\section{Results}

The flow diagram is presented in Fig. 1. A total of 35 diagnostic studies were identified, assessed and interpreted. The characteristics of these studies are presented in Table 3. 20 studies were performed in an emergency department, four studies in a traumatology setting and three other studies in a radiology department. The patients in the studies by Mallee et al. [56-58] were derived from one prospective study; therefore the setting was the same for each study: patients were initially seen by the emergency physicians and in follow-up by the orthopaedic department and/or trauma surgery department, depending on who was on call. In five studies the setting was not specified. To our knowledge, all first authors of those five studies were working in a hospital care setting, so we assume all to have been done in hospital care. History taking, physical examination and imaging as index tests were investigated in $0,20 \%(7 / 35)$ $[48,53,62,64,67,73,77]$ and $86 \%(30 / 35)$ [43-47, 49$51,53-61,63,65,66,68-77]$ of the studies, respectively.

\section{Quality assessment}

There was considerable underreporting of important quality domains in 23 of the 35 studies (see Table 4). In 13 of the 35 studies [43, 44, 48, 50, 54, 55, 59, 64, 67, 72, $74,76,77]$, patient selection was not well documented. Furthermore, the risk of bias was predominantly due to the absence of a proper description of the index test (9/ 35) $[43,45,49,53,55,64,65,72,77]$ or the reference standard $(13 / 35)[45,49,55,62,64-68,71-73,75]$. 
Table 2 Example electronic search strategy

Database Search terms

Embase ('hand injury'/exp. OR 'wrist injury'/exp. OR 'wrist fracture'/exp. OR (('hand bone'/exp. OR wrist/exp. OR hand/exp. OR 'wrist pain'/exp. OR 'hand pain'/exp) AND ('bone injury'/exp. OR fracture/de OR 'ligament injury'/exp. OR 'ligament rupture'/exp)) OR (((hand OR hands OR wrist* OR finger* OR carpal* OR carpus OR phalanx* OR metacarp* OR capitate* OR hamat* OR lunat* OR pisiform* OR scaphoid* OR trapezium* OR trapezoid* OR triquetr* OR navicular* OR lunar OR semilunar* OR multangulum* OR pyramid* OR metacarpophalang* OR thumb* OR 'distal radius' OR 'distal ulna' OR 'distal radial' OR 'distal ulnar' OR scapholunate* OR lunotriquetral* OR 'triangular fibrocartilaginous' OR SLIL OR LTIL OR tfcc OR 'ulnar collateral ligament' OR 'ulnar collateral ligaments' OR ucl) NEAR/3 (injur* OR trauma* OR wound* OR lesion* OR dislocate* OR fracture* OR damage* OR tear* OR sprain* OR displace* OR rupture*))):ab,ti) AND ('diagnostic test'/de OR 'function test'/exp. OR 'diagnostic error'/exp. OR 'diagnostic accuracy'/exp. OR 'diagnostic value'/exp. OR 'differential diagnosis'/ exp. OR 'delayed diagnosis'/exp. OR 'sensitivity and specificity'/exp. OR (((diagnos* OR detect* OR differen* OR strength* OR motion*) NEAR/3 (test* OR accura* OR error* OR false OR fail* OR value* OR impact* OR effective* OR earl* OR missed OR correct* OR incorrect* OR delay* OR difficult* OR negative* OR positive* OR sensitivit* OR specificit* OR confirm* OR abilit*)) OR (diagnos* NEAR/3 differen*) OR misdiagnos* OR underdiagnos* OR undetect* OR (predict* NEAR/3 value*) OR (function* NEAR/3 test*) OR (false NEAR/3 (negative* OR positive*)):ab,ti) NOT ([Conference Abstract]/lim OR [Letter]/lim OR [Note]/lim OR [Editorial]/lim) AND [english]/lim NOT ([animals]/lim NOT [humans]/lim)

Search terms for the other databases are available upon request

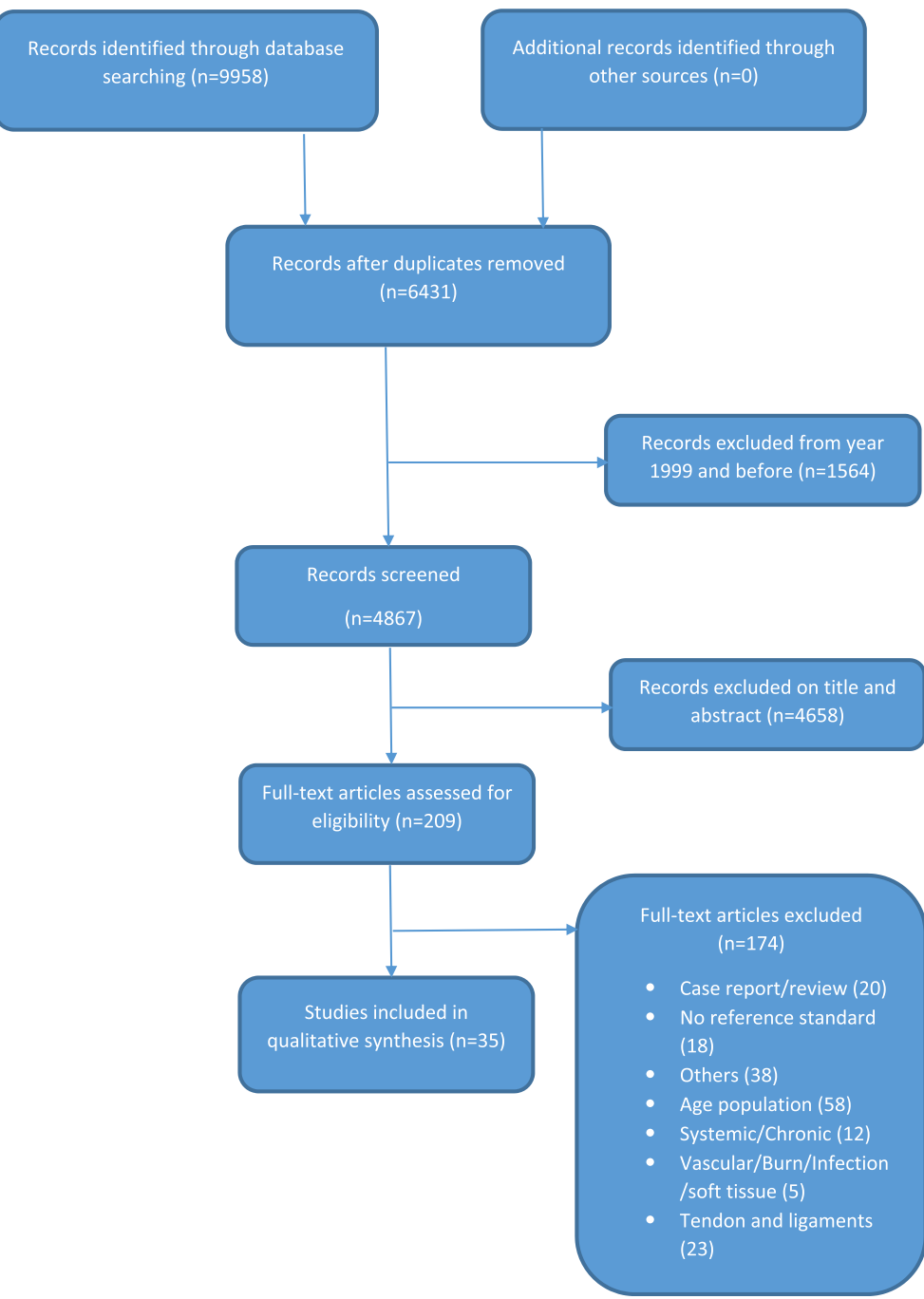

Fig. 1 Flow chart study selection 
Table 3 Characteristics of the Eligible Studies $(N=35)$

\begin{tabular}{|c|c|c|c|c|c|c|}
\hline Author(s) & Participants & Design & $\begin{array}{l}\text { Department of patient } \\
\text { presentation (Country) }\end{array}$ & Fracture & Index test & Reference test \\
\hline \multicolumn{7}{|c|}{ SCAPHOID AND OTHER CARPAL BONES FRACTURES } \\
\hline $\begin{array}{l}\text { Adey } \\
\text { (2007) [43] }\end{array}$ & 30 & Retrospective & Not described (USA) & Scaphoid & CT & $\begin{array}{l}\text { Radiographs } 6 \text { weeks after } \\
\text { injury }\end{array}$ \\
\hline $\begin{array}{l}\text { Annamalai } \\
\text { (2003) [44] }\end{array}$ & 50 & Retrospective & Not described (Scotland) & Scaphoid & $\begin{array}{l}\text { Radiology (scaphoid } \\
\text { and pronator fat } \\
\text { stripe) }\end{array}$ & MRI 0,2 T (12-72 h) \\
\hline $\begin{array}{l}\text { Behzadi } \\
\text { (2015) [45] }\end{array}$ & 124 & Retrospective & $\begin{array}{l}\text { Emergency department } \\
\text { (Germany) }\end{array}$ & Scaphoid & $\begin{array}{l}\text { Radiographs (anterior- } \\
\text { posterior, lateral and } \\
\text { oblique projections) }\end{array}$ & MDCT (within 10 days) \\
\hline $\begin{array}{l}\text { Beeres } \\
(2007)[46]\end{array}$ & 50 & Prospective & $\begin{array}{l}\text { Emergency department } \\
\text { (Netherlands) }\end{array}$ & $\begin{array}{l}\text { Scaphoid } \\
\text { and other } \\
\text { carpal bones }\end{array}$ & $\begin{array}{l}\text { Bone scintigraphy } \\
\text { (3-7 days after injury) }\end{array}$ & $\begin{array}{l}\text { Clinical outcome: physical } \\
\text { examination at fixed } \\
\text { intervals } \\
\text { No fracture, with a } \\
\text { normal physical } \\
\text { examination at } 2 \text { or } 6 \\
\text { weeks, BS was considered } \\
\text { correct. However, if there } \\
\text { were clinical signs of a } \\
\text { fracture after } 2 \text { and } 6 \\
\text { weeks, BS was considered } \\
\text { false negative. } \\
\text { Another fracture in the } \\
\text { carpal region and } \\
\text { physical examination after } \\
2 \text { weeks (during change } \\
\text { of cast) matched with } \\
\text { such a fracture, BS was } \\
\text { considered correct. But, } \\
\text { when physical } \\
\text { examination after } 2 \text { weeks } \\
\text { showed no signs of } \\
\text { fracture, BS was } \\
\text { considered false positive. } \\
\text { A scaphoid fracture, } \\
\text { confirmed on physical } \\
\text { examination after } 2 \text { weeks } \\
\text { (during change of cast), } \\
\text { BS was considered } \\
\text { correct. If however, } \\
\text { neither physical } \\
\text { examination after } 2 \\
\text { weeks, nor consecutive } \\
\text { physical examinations } \\
\text { showed evidence of a } \\
\text { scaphoid fracture, there } \\
\text { was no scaphoid fracture. } \\
\text { BS was then considered } \\
\text { false positive. }\end{array}$ \\
\hline $\begin{array}{l}\text { Beeres } \\
(2008)[47]\end{array}$ & 100 & Prospective & $\begin{array}{l}\text { Emergency department } \\
\text { (Netherlands) }\end{array}$ & Scaphoid & $\begin{array}{l}\text { MRI } 1.5 \mathrm{~T}(<24 \mathrm{~h}) \text { and } \\
\text { Bone scintigraphy } \\
\text { (between } 3 \text { and } 5 \\
\text { days) }\end{array}$ & $\begin{array}{l}\text { Absence or presence of a } \\
\text { fracture on both MRI } \\
\text { and bone scintigraphy, } \\
\text { or in the case of } \\
\text { discrepancy, clinical } \\
\text { and/or radiological } \\
\text { evidence of a fracture. }\end{array}$ \\
\hline $\begin{array}{l}\text { Bergh } \\
(2014)[48]\end{array}$ & 154 & Prospective & $\begin{array}{l}\text { Emergency department, } \\
\text { outpatient clinic (Norway) }\end{array}$ & Scaphoid & $\begin{array}{l}\text { Clinical Scaphoid } \\
\text { Score (CSS): } \\
\text { tenderness in the } \\
\text { anatomical snuffbox } \\
\text { with the wrist in ulnar } \\
\text { deviation ( } 3 \text { points) + } \\
\text { tenderness over the } \\
\text { scaphoid tubercle ( } 2 \\
\text { points) + pain upon }\end{array}$ & MRI $1.5 \mathrm{~T}$ \\
\hline
\end{tabular}


Table 3 Characteristics of the Eligible Studies $(N=35)$ (Continued)

\begin{tabular}{|c|c|c|c|c|c|c|}
\hline Author(s) & Participants & Design & $\begin{array}{l}\text { Department of patient } \\
\text { presentation (Country) }\end{array}$ & Fracture & Index test & Reference test \\
\hline & & & & & $\begin{array}{l}\text { longitudinal } \\
\text { compression of the } \\
\text { thumb ( } 1 \text { point })\end{array}$ & \\
\hline $\begin{array}{l}\text { Breederveld } \\
\text { (2004) [49] }\end{array}$ & 29 & Prospective & $\begin{array}{l}\text { Emergency department } \\
\text { (Netherlands) }\end{array}$ & Scaphoid & BS (three-fase) and CT & $\begin{array}{l}\text { Clinical follow-up } \\
\text { (including CT and } \\
\text { Bone scintigraphy) }\end{array}$ \\
\hline $\begin{array}{l}\text { Cruickshank } \\
\text { (2007) [50] }\end{array}$ & 47 & Prospective & $\begin{array}{l}\text { Teaching emergency } \\
\text { department (Australia) }\end{array}$ & $\begin{array}{l}\text { Scaphoid } \\
\text { and other } \\
\text { carpal bones }\end{array}$ & CT (same or next day) & $\begin{array}{l}\text { The diagnosis on Day } \\
10 \text { with clinical } \\
\text { examination and } \\
\text { X-rays, with MRI } \\
\text { performed in patients } \\
\text { with persistent } \\
\text { tenderness but } \\
\text { normal X-rays. }\end{array}$ \\
\hline $\begin{array}{l}\text { Fusetti } \\
(2005)[51]\end{array}$ & 24 & Prospective & Not described (Switzerland) & Scaphoid & $\begin{array}{l}\text { HSR-S }(<24 \mathrm{~h} \text { of the } \\
\text { clinical examination) }\end{array}$ & $\begin{array}{l}\text { CT (immediately after } \\
\text { HSR-S performed) }\end{array}$ \\
\hline $\begin{array}{l}\text { Gabler (2001) } \\
\text { [52] }\end{array}$ & 121 & Prospective & $\begin{array}{l}\text { Department of traumatology: } \\
\text { fracture clinics (Austria) }\end{array}$ & Scaphoid & $\begin{array}{l}\text { Repeated clinical } \\
\text { examination } \\
\text { (tenderness over the } \\
\text { anatomical snuff box } \\
\text { or the carpus as well } \\
\text { as a positive scaphoid } \\
\text { compression test) and } \\
\text { radiological } \\
\text { examinations } \\
\text { (scaphoid views) }\end{array}$ & MRI $1.0 \mathrm{~T}$ \\
\hline $\begin{array}{l}\text { Herneth } \\
\text { (2001) [53] }\end{array}$ & 15 & Prospective & Not described (Austria) & Scaphoid & $\begin{array}{l}\text { Clinical examination, } \\
\text { radiography and } \\
\text { High-spatial resolution } \\
\text { ultrasonography }\end{array}$ & MRI 1,0T $(<72 \mathrm{~h})$ \\
\hline $\begin{array}{l}\text { Ilica (2011) } \\
{[54]}\end{array}$ & 54 & Prospective & Emergency department (Turkey) & Scaphoid & $\mathrm{MDCT}$ & MRI $1.5 \mathrm{~T}$ \\
\hline $\begin{array}{l}\text { Kumar } \\
\text { (2005) [55] }\end{array}$ & 22 & Prospective & $\begin{array}{l}\text { Collaboration between the } \\
\text { Department of Emergency } \\
\text { Medicine and Medical Imaging } \\
\text { (New Zealand) }\end{array}$ & Scaphoid & MRI $1.5 \mathrm{~T}(<24 \mathrm{~h})$ & $\begin{array}{l}\text { MRI in those without } \\
\text { fracture at MRI }<24 \mathrm{~h} \\
\text { or no clinical signs of } \\
\text { fracture }\end{array}$ \\
\hline $\begin{array}{l}\text { Mallee } \\
(2011)[56]\end{array}$ & 34 & Prospective & $\begin{array}{l}\text { Initially emergency physicians } \\
\text { and in follow-up by the } \\
\text { Orthopedic department } \\
\text { and/or Trauma surgery } \\
\text { department, depending } \\
\text { on who was on call. } \\
\text { (Netherlands) }\end{array}$ & Scaphoid & $\begin{array}{l}\mathrm{CT} \text { and MRI } 1.0 \mathrm{~T} \\
\text { (within } 10 \text { days) }\end{array}$ & $\begin{array}{l}\text { Radiographs, after } 6 \\
\text { weeks follow-up }\end{array}$ \\
\hline $\begin{array}{l}\text { Mallee } \\
\text { (2016) [57] }\end{array}$ & 34 & Prospective & $\begin{array}{l}\text { Initially emergency physicians } \\
\text { and in follow-up by the } \\
\text { Orthopedic department and/ } \\
\text { or Trauma surgery department, } \\
\text { depending on who was on call. } \\
\text { (Netherlands) }\end{array}$ & Scaphoid & $\begin{array}{l}\text { 6-weeks radiographs } \\
\text { in JPEG- and DICOM- } \\
\text { view }\end{array}$ & $C T, M R I$, or $C T$ and $M R I$ \\
\hline $\begin{array}{l}\text { Mallee } \\
\text { (2014) [58] }\end{array}$ & 34 & Prospective & $\begin{array}{l}\text { Initially emergency physicians } \\
\text { and in follow-up by the } \\
\text { Orthopedic department and/ } \\
\text { or Trauma surgery department, } \\
\text { depending on who was on call. } \\
\text { (Netherlands) }\end{array}$ & Scaphoid & $\begin{array}{l}\text { CT-scaphoid: } \\
\text { reformations in planes } \\
\text { defined by the long } \\
\text { axis of the scaphoid. } \\
\text { CT-wrist: reformations } \\
\text { made in the anatomic } \\
\text { planes of the wrist. } \\
\text { CT performed within } \\
10 \text { days. }\end{array}$ & $\begin{array}{l}\text { Radiographs in four } \\
\text { standard scaphoid views } \\
\text { after } 6 \text { weeks follow-up. }\end{array}$ \\
\hline $\begin{array}{l}\text { Memarsadeghi } \\
\text { (2006) [59] }\end{array}$ & 29 & Prospective & Not described (Austria) & Scaphoid & MDCT and MRI 1,0 T & $\begin{array}{l}\text { Radiographs obtained } 6 \\
\text { weeks after trauma. View: } \\
\text { posteroanterior with the }\end{array}$ \\
\hline
\end{tabular}


Table 3 Characteristics of the Eligible Studies $(N=35)$ (Continued)

\begin{tabular}{|c|c|c|c|c|c|}
\hline Author(s) & Participants & Design & $\begin{array}{l}\text { Department of patient } \\
\text { presentation (Country) }\end{array}$ & Fracture & Index test \\
\hline $\begin{array}{l}\text { Ottenin } \\
\text { (2012) [60] }\end{array}$ & 100 & Retrospective & $\begin{array}{l}\text { Radiology department of the } \\
\text { emergency unit (France) }\end{array}$ & $\begin{array}{l}\text { Scaphoid } \\
\text { and other } \\
\text { carpal bones }\end{array}$ & $\begin{array}{l}\text { Tomosynthesis } \\
\text { (frontal and lateral), } \\
\text { MDCT (within } 7 \text { days) } \\
\text { and radiographs } \\
\text { (posteroanterior } \\
\text { view, lateral view, } \\
\text { anteroposterior } \\
\text { oblique view, } \\
\text { scaphoid view with } \\
\text { ulnar deviation, and } \\
\text { posteroanterior view } \\
\text { with clenched fist) }\end{array}$ \\
\hline
\end{tabular}

Reference test

wrist in neutral position,

lateral, semipronated

oblique scaphoid, and

radial oblique scaphoid.

The reference standard

for each case was

determined after

completion of all

examinations; analysis of MRI $(n=13$; performed in cases of doubt after completion of diagnostic standard radiography, tomosynthesis, and (T); and follow-up information obtained by physical examination or, in case of no clinical follow-up, by telephone recalls.

Platon

(2011) [61]

62

Prospective

Emergency department (Switzerland)

Scaphoid

US within 3 days (presence of a cortical interruption of the scaphoid along with a radio-carpal or scaphotrapeziumtrapezoid effusion)

Emergency department (Netherlands)
$\operatorname{MDCT}(<24 \mathrm{~h})$ and Bone scintigraphy (3-5 days)
CT (immediately after US)

Final diagnosis after final discharge, according to the following standard:

If $\mathrm{CT}$ and bone scintigraphy showed a fracture, the final diagnosis was fracture. If $\mathrm{CT}$ and bone scintigraphy showed no fracture, the final diagnosis was no fracture. In case of discrepancy between $\mathrm{CT}$ and bone scintigraphy, both radiographic (6 weeks after injury) and physical reevaluation during follow-up were used to make a final diagnosis. In case of radiographic evidence of a scaphoid fracture 6 weeks after injury, the final diagnosis was fracture. In case of no radiographic evidence of a scaphoid fracture 6 weeks after injury but there were persistent clinical signs of a scaphoid fracture after 2 weeks, the final diagnosis was fracture.

If there was no radiographic evidence of a scaphoid fracture 6 weeks after injury and there were no longer clinical signs of a 
Table 3 Characteristics of the Eligible Studies $(N=35)$ (Continued)

\begin{tabular}{|c|c|c|c|c|c|c|}
\hline Author(s) & Participants & Design & $\begin{array}{l}\text { Department of patient } \\
\text { presentation (Country) }\end{array}$ & Fracture & Index test & Reference test \\
\hline & & & & & & $\begin{array}{l}\text { scaphoid fractures } \\
\text { throughout follow-up, the } \\
\text { final diagnosis was no } \\
\text { fracture. }\end{array}$ \\
\hline $\begin{array}{l}\text { Rhemrev } \\
\text { (2010) [63] }\end{array}$ & 78 & Prospective & $\begin{array}{l}\text { Emergency department } \\
\text { (Netherlands) }\end{array}$ & Scaphoid & $\begin{array}{l}\text { Three clinical exams: } \\
\text { 1) inspection of the } \\
\text { snuffbox for the } \\
\text { presence of } \\
\text { ecchymosis or } \\
\text { edema, 2) flexion and } \\
\text { extension of the wrist, } \\
\text { 3) Supination and } \\
\text { pronation strength, 4) } \\
\text { Grip strength. }\end{array}$ & $\begin{array}{l}\text { MRI 1,5 T, bone } \\
\text { scintigraphy, radiography } \\
\text { and physical re-evaluation } \\
\text { during } 6 \text { weeks clinical } \\
\text { follow-up. }\end{array}$ \\
\hline $\begin{array}{l}\text { Steenvoorde } \\
\text { (2006) [64] }\end{array}$ & 31 & $\begin{array}{l}\text { Not } \\
\text { described }\end{array}$ & $\begin{array}{l}\text { Emergency department } \\
\text { (Netherlands): request for } \\
\text { radiograph of the scaphoid } \\
\text { by general practitioners were } \\
\text { excluded }\end{array}$ & $\begin{array}{l}\text { Scaphoid } \\
\text { and other } \\
\text { carpal bones }\end{array}$ & $\begin{array}{l}\text { Five or more positive } \\
\text { clinical tests out of } \\
\text { seven tests: 1) loss of } \\
\text { concavity of the } \\
\text { anatomic snuff box, 2) } \\
\text { snuffbox tenderness, } \\
\text { 3) the clamp sign, 4) } \\
\text { palmar tenderness of } \\
\text { the scaphoid, 5) axial } \\
\text { compression of the } \\
\text { thumb along its } \\
\text { longitudinal axis, } \\
\text { 6) site of pain on } \\
\text { resisted supination, 7) } \\
\text { site of pain on ulnar } \\
\text { deviation. }\end{array}$ & Clinical follow-up \\
\hline $\begin{array}{l}\text { Yildirim } \\
\text { (2013) [65] }\end{array}$ & 63 & Prospective & $\begin{array}{l}\text { Emergency department } \\
\text { (Turkey) }\end{array}$ & Scaphoid & $\begin{array}{l}\text { BUS (presence of a } \\
\text { cortical interruption } \\
\text { of the scaphoid along } \\
\text { with a radiocarpal or } \\
\text { scaphotrapezium } \\
\text { trapezoid effusion) }\end{array}$ & MRI (<24h) \\
\hline $\begin{array}{l}\text { de Zwart } \\
\text { (2016) [66] }\end{array}$ & 33 & Prospective & $\begin{array}{l}\text { Emergency department } \\
\text { (Netherlands) }\end{array}$ & Scaphoid & $\begin{array}{l}\mathrm{MRI}(<72 \mathrm{~h}), \mathrm{CT}(< \\
72 \mathrm{~h}) \text { and Bone } \\
\text { Scintigraphy } \\
\text { (between } 3 \text { and } 5 \\
\text { days) }\end{array}$ & $\begin{array}{l}\text { If MRI, CT and BS all } \\
\text { showed a fracture, the } \\
\text { final diagnosis was: } \\
\text { fracture. } \\
\text { If MRI, } \mathrm{CT} \text { and BS all } \\
\text { showed no fracture, the } \\
\text { final diagnosis was: no } \\
\text { fracture. } \\
\text { In case of discrepancy } \\
\text { between MRI, CT and BS, } \\
\text { the final diagnosis was } \\
\text { established based on } \\
\text { specific clinical } \\
\text { signs of a fracture after } 6 \\
\text { weeks (tender anatomic } \\
\text { snuffbox and pain in the } \\
\text { snuffbox when applying } \\
\text { axial pressure on the first } \\
\text { or second digit) } \\
\text { combined with the } \\
\text { radiographic evidence of } \\
\text { a fracture after } 6 \text { weeks. If } \\
\text { these signs were absent } \\
\text { and no radiographic } \\
\text { evidence, the final } \\
\text { diagnosis was: no } \\
\text { fracture. }\end{array}$ \\
\hline Sharifi & 175 & Prospective & Emergency department & Scaphoid & VAS pain score & MRI \\
\hline
\end{tabular}


Table 3 Characteristics of the Eligible Studies $(N=35)$ (Continued)

\begin{tabular}{|c|c|c|c|c|c|c|}
\hline Author(s) & Participants & Design & $\begin{array}{l}\text { Department of patient } \\
\text { presentation (Country) }\end{array}$ & Fracture & Index test & Reference test \\
\hline (2015) [67] & & & (Iran) & fractures & $\begin{array}{l}\text { (anatomical snuff } \\
\text { box tenderness) }\end{array}$ & \\
\hline $\begin{array}{l}\text { Brink } \\
(2014)[68]\end{array}$ & 98 & Prospective & $\begin{array}{l}\text { Department of Radiology } \\
\text { (Netherlands) }\end{array}$ & $\begin{array}{l}\text { Fractures } \\
\text { carpus and } \\
\text { metacarpal }\end{array}$ & $\mathrm{CT}$ or radiography & Clinical follow-up \\
\hline $\begin{array}{l}\text { Neubauer } \\
\text { (2018) [69] }\end{array}$ & 102 & Retrospective & $\begin{array}{l}\text { Orthopedics and Trauma/ } \\
\text { Hand Surgery (Germany) }\end{array}$ & $\begin{array}{l}\text { Scaphoid } \\
\text { fractures }\end{array}$ & CBCT or radiography & $\begin{array}{l}\text { Clinical follow-up } \\
\text { (including images) }\end{array}$ \\
\hline $\begin{array}{l}\text { Borel } \\
(2017)[70]\end{array}$ & 49 & Prospective & $\begin{array}{l}\text { Orthopedics and Trauma } \\
\text { Surgery (France) }\end{array}$ & $\begin{array}{l}\text { Scaphoid } \\
\text { or wrist } \\
\text { fractures }\end{array}$ & CBCT & MRI \\
\hline \multicolumn{7}{|c|}{ SCAPHOID, OTHER CARPAL AND METACARPAL BONES FRACTURES } \\
\hline $\begin{array}{l}\text { Balci } \\
(2015)[71]\end{array}$ & 455 & Retrospective & $\begin{array}{l}\text { Emergency department } \\
\text { (Turkey) }\end{array}$ & $\begin{array}{l}\text { Carpal and } \\
\text { metacarpal }\end{array}$ & Radiographs & MDCT \\
\hline $\begin{array}{l}\text { Jorgsholm } \\
\text { (2013) [72] }\end{array}$ & 296 & Prospective & $\begin{array}{l}\text { Emergency department } \\
\text { (Sweden) }\end{array}$ & $\begin{array}{l}\text { Scaphoid, } \\
\text { other } \\
\text { carpal and } \\
\text { metacarpal } \\
\text { bones }\end{array}$ & $\begin{array}{l}\text { Radiographs } \\
\text { (dorsovolar and } \\
\text { lateral projections } \\
\text { with an additional } \\
4 \text { views of the } \\
\text { scaphoid.) and } C \top\end{array}$ & $\begin{array}{l}\text { MRI } 0.23 \mathrm{~T} \text { (within } \\
3 \text { days) }\end{array}$ \\
\hline $\begin{array}{l}\text { Nikken } \\
\text { (2005) [73] }\end{array}$ & 87 & Prospective & $\begin{array}{l}\text { Radiology department referred } \\
\text { by traumatologist, orthopedic } \\
\text { surgeon or emergency } \\
\text { physician (Netherlands) }\end{array}$ & $\begin{array}{l}\text { Scaphoid } \\
\text { and other } \\
\text { carpal bones. } \\
\text { Metacarpal } \\
\text { bones II-IV }\end{array}$ & $\begin{array}{l}\text { Anatomic snuffbox } \\
\text { tenderness, } \\
\text { radiographs } \\
\text { (posteroanterior } \\
\text { and lateral projection) } \\
\text { and MRI 0,2T (short } \\
\text { procedure) }\end{array}$ & Additional treatment \\
\hline \multicolumn{7}{|c|}{ CARPAL AND METACARPAL BONES AND PHALANGEAL FRACTURES } \\
\hline $\begin{array}{l}\text { Javadzadeh } \\
\text { (2014) [74] }\end{array}$ & 260 & $\begin{array}{l}\text { Not } \\
\text { described }\end{array}$ & Emergency department (Iran) & $\begin{array}{l}\text { Carpal, } \\
\text { metacarpal, } \\
\text { and phalangeal }\end{array}$ & $\begin{array}{l}\text { BUS and WBT } \\
\text { ultrasonography }\end{array}$ & $\begin{array}{l}\text { Radiographs (not } \\
\text { described when } \\
\text { performed) }\end{array}$ \\
\hline \multicolumn{7}{|c|}{ METACARPAL BONES AND/OR PHALANGEAL FRACTURES } \\
\hline $\begin{array}{l}\text { Faccioli } \\
\text { (2010) [75] }\end{array}$ & 57 & Prospective & $\begin{array}{l}\text { Traumatology department } \\
\text { (Italy) }\end{array}$ & Phalangeal & CBCT & MSCT \\
\hline $\begin{array}{l}\text { Kocaoglu } \\
(2016)[76]\end{array}$ & 96 & Prospective & Emergency department (Turkey) & Metacarpal & US & $\begin{array}{l}\text { Radiographs } \\
\text { (anteroposterior } \\
\text { and oblique) }\end{array}$ \\
\hline $\begin{array}{l}\text { Tayal } \\
\text { (2007) [77] }\end{array}$ & 78 & Prospective & Emergency department (USA) & $\begin{array}{l}\text { Metacarpal and } \\
\text { phalangeal }\end{array}$ & $\begin{array}{l}\text { US and physical } \\
\text { examination }\end{array}$ & $\begin{array}{l}\text { Radiographs and } \\
\text { when operated, } \\
\text { surgical findings }\end{array}$ \\
\hline
\end{tabular}

MRI Magnetic resonance imaging, CT Computed Tomography, CBCT Cone Beam Computed Tomography, MSCT Multi-slice Computed Tomography, HSR-S High Spatial Resolution sonography, BUS Bedside ultrasonography, WBT Water bath technique ROM Range of motion

Twelve of the studies (34\%) demonstrated no limitations when risk of bias was assessed, according to QUADAS-2 $[46,47,51,52,56-58,60,61,63,69,70]$. Eight showed incorporation bias $[46,47,49,55,60,62,66,69]$.

\section{Diagnosing carpal fractures in hospital care}

Table 5 presents the accuracy of the diagnostic tests of all the carpal fractures. Two studies described the diagnostic accuracy of history taking [62, 67]. Physical examination $[48,53,62,64]$ and combined physical and radiological examination [52] for diagnosing scaphoid fractures showed Se, Sp, accuracy, PPV and NPV ranging from 15 to $100 \%, 13-98 \%, 55-73 \%, 14-73 \%$ and $75-100 \%$, respectively.

Repeated physical examination with radiological examination after 38 days [52] for diagnosing other carpal bone fractures showed a Se of $100 \%$ with the exception of the triquetrum $(75 \%)$.

Radiographs used as an index test for diagnosing scaphoid fractures showed Se, Sp, accuracy, PPV and NPV ranging from 25 to $87 \%, 50-100 \%, 48-88 \%, 14-100 \%$ and 49-94\%, respectively. For diagnosing scaphoid fractures, Magnetic Resonance Imaging (MRI) as an imaging modality showed Se, Sp, accuracy, PPV and NPV ranging from 67 to $100 \%, 89-100 \%, 85-100 \%, 54-100 \%$ and $93-100 \%$, 
Table 4 Summary of Methodological Quality according to Quality Assessment of Diagnostic Accuracy Studies-2

\begin{tabular}{|c|c|c|c|c|c|c|c|}
\hline \multirow[t]{2}{*}{ Author(s) } & \multicolumn{4}{|l|}{ Risk of Bias } & \multicolumn{3}{|c|}{ Applicability Concerns } \\
\hline & Patient Selection & Index Test & Reference standard & Flow and Timing & Patient Selection & Index Test & Reference standard \\
\hline Adey (2007) [43] & HR & $U R$ & LR & LR & LR & $L R$ & $L R$ \\
\hline Annamalai (2003) [44] & $H R$ & LR & LR & LR & $L R$ & LR & $L R$ \\
\hline Balci (2015) [71] & $L R$ & LR & HR & $L R$ & $L R$ & LR & $L R$ \\
\hline Beeres (2007) [46] & $L R$ & LR & LR & LR & $L R$ & LR & $L R$ \\
\hline Beeres (2008) [47] & $L R$ & $L R$ & $L R$ & $L R$ & LR & LR & $L R$ \\
\hline Behzadi (2015) [45] & $L R$ & HR & HR & LR & $L R$ & $L R$ & $L R$ \\
\hline Bergh (2014) [48] & $U R$ & $L R$ & $L R$ & $L R$ & $L R$ & $L R$ & $L R$ \\
\hline Borel (2017) [70] & $L R$ & $L R$ & $L R$ & $L R$ & $L R$ & $L R$ & $L R$ \\
\hline Breederveld (2004) [49] & $L R$ & $U R$ & $U R$ & $L R$ & $L R$ & LR & $L R$ \\
\hline Brink (2019) [68] & $L R$ & $L R$ & HR & $L R$ & $L R$ & $L R$ & $L R$ \\
\hline Cruickshank (2007) [50] & $U R$ & LR & LR & LR & $L R$ & LR & $L R$ \\
\hline Faccioli (2010) [75] & $L R$ & HR & HR & $L R$ & $L R$ & $L R$ & $L R$ \\
\hline Fusetti (2005) [51] & $L R$ & LR & $L R$ & $L R$ & $L R$ & $L R$ & $L R$ \\
\hline Gabler (2001) [52] & $L R$ & $L R$ & $L R$ & $L R$ & $L R$ & $L R$ & $L R$ \\
\hline Herneth (2001) [53] & $L R$ & $U R$ & $L R$ & $L R$ & $L R$ & LR & $L R$ \\
\hline Ilica (2011) [54] & $U R$ & LR & $L R$ & $L R$ & $L R$ & LR & $L R$ \\
\hline Javadzadeh (2014) [74] & $U R$ & LR & LR & $L R$ & $L R$ & LR & $L R$ \\
\hline Jorgsholm (2013) [72] & $U R$ & HR & HR & $L R$ & $L R$ & $L R$ & $L R$ \\
\hline Kocaoglu (2016) [76] & $U R$ & $L R$ & $L R$ & $L R$ & $L R$ & $L R$ & $L R$ \\
\hline Kumar (2005) [55] & $U R$ & HR & HR & HR & $L R$ & $L R$ & $L R$ \\
\hline Mallee (2011) [56] & $L R$ & $L R$ & LR & LR & $L R$ & $L R$ & $L R$ \\
\hline Mallee (2016) [57] & $L R$ & $L R$ & $L R$ & $L R$ & $L R$ & $L R$ & $L R$ \\
\hline Mallee (2014) [58] & LR & $L R$ & LR & $L R$ & $L R$ & LR & $L R$ \\
\hline Memarsadeghi (2006) [59] & $U R$ & LR & LR & $L R$ & $L R$ & $L R$ & $L R$ \\
\hline Neubauer (2018) [69] & $L R$ & LR & LR & LR & $L R$ & LR & $L R$ \\
\hline Nikken (2005) [73] & $L R$ & $L R$ & HR & $L R$ & $L R$ & $L R$ & $L R$ \\
\hline Ottenin (2012) [60] & $L R$ & $L R$ & LR & $L R$ & $L R$ & $L R$ & $L R$ \\
\hline Platon (2011) [61] & $L R$ & $L R$ & $L R$ & $L R$ & $L R$ & $L R$ & $L R$ \\
\hline Rhemrev (2010) [62] & $L R$ & $L R$ & HR & $L R$ & $L R$ & $L R$ & $L R$ \\
\hline Rhemrev (2010) [63] & $L R$ & $L R$ & LR & LR & $L R$ & LR & $L R$ \\
\hline Sharifi (2015) [67] & $U R$ & $L R$ & $U R$ & $L R$ & $L R$ & $L R$ & $L R$ \\
\hline Steenvoorde (2006) [64] & $U R$ & HR & HR & $L R$ & $L R$ & $L R$ & $L R$ \\
\hline Tayal (2007) [77] & $U R$ & $L R$ & $L R$ & $L R$ & $L R$ & $L R$ & $L R$ \\
\hline Yildirim (2013) [65] & $L R$ & HR & HR & HR & $L R$ & LR & $L R$ \\
\hline de Zwart (2016) [66] & $L R$ & LR & HR & LR & $L R$ & $L R$ & $L R$ \\
\hline
\end{tabular}

Abbreviations: LR Low Risk, HR High Risk, UR Unclear Risk

respectively. Multi Detector Computed Tomography (MDCT) showed Se, Sp, accuracy, PPV and NPV ranging from 33 to $100 \%, 85-100 \%, 79-100 \%, 28-100 \%$ and $86-$ $100 \%$, respectively. Bone Scintigraphy (BS) as an index test for diagnosing scaphoid fractures showed Se, Sp, accuracy, PPV and NPV ranging from 78 to $100 \%, 87-97 \%$, 86$97 \%, 62-78 \%$ and $90-100 \%$, respectively. For diagnosing scaphoid fractures, Ultrasonography (US) as an imaging modality showed Se, Sp, accuracy, PPV and NPV ranging from 78 to $100 \%, 34-100 \%, 49-100 \%, 30-100 \%$ and $75-$ $100 \%$, respectively.

\section{Diagnosing phalangeal and metacarpal fractures in hospital care}

Table 5 also presents the accuracy of the diagnostic tests for metacarpal and/or phalangeal fractures, as described 


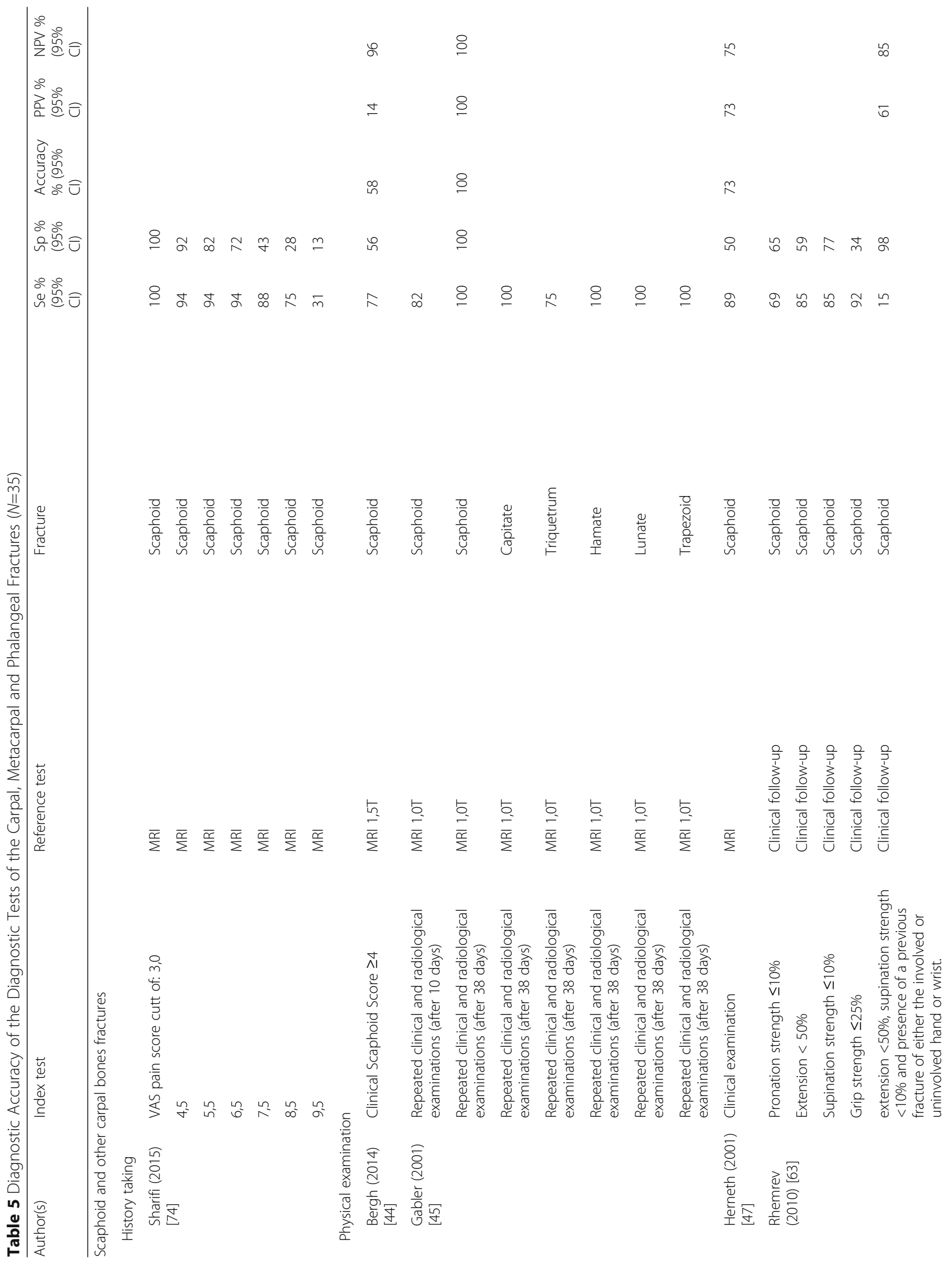




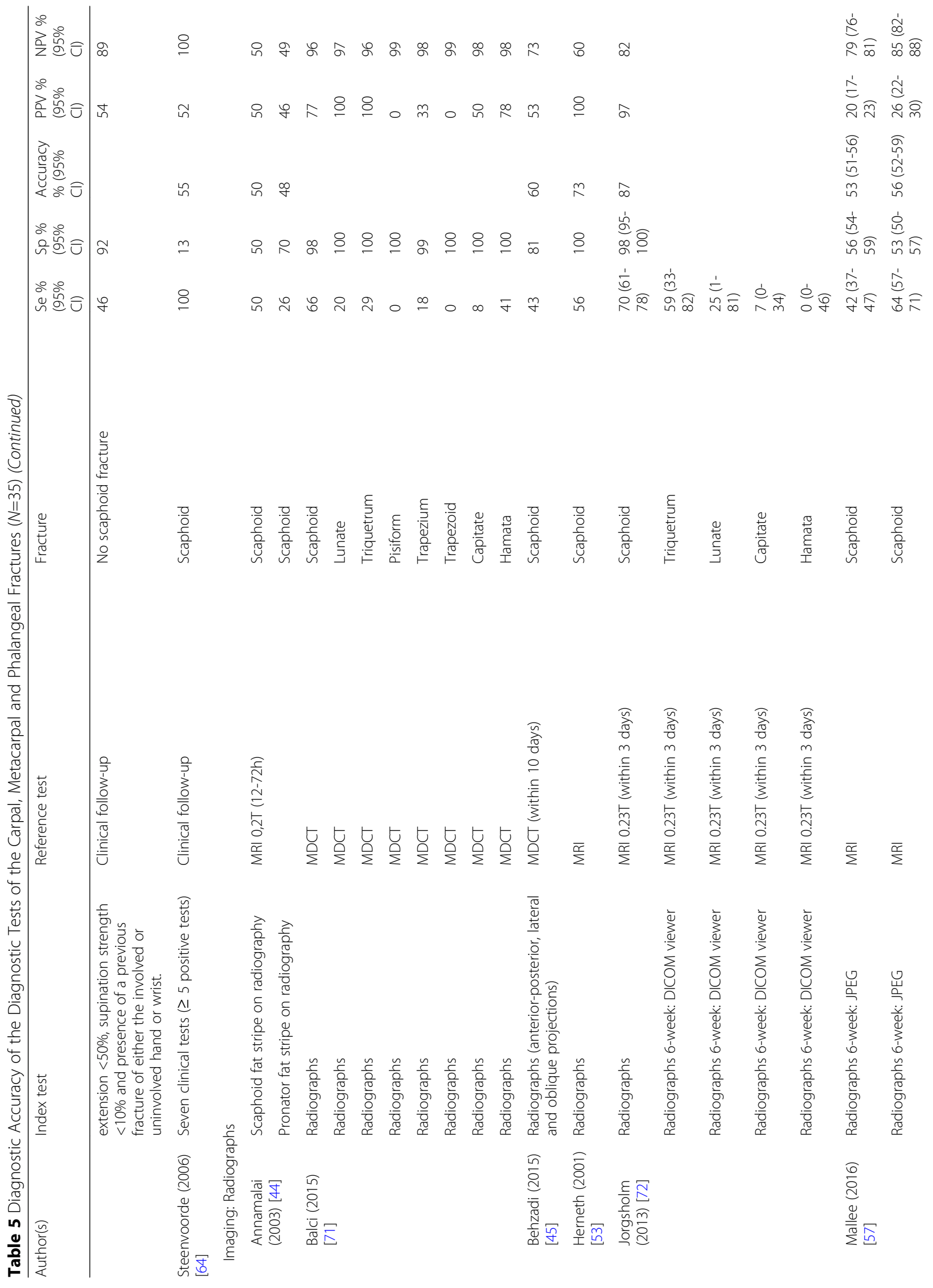




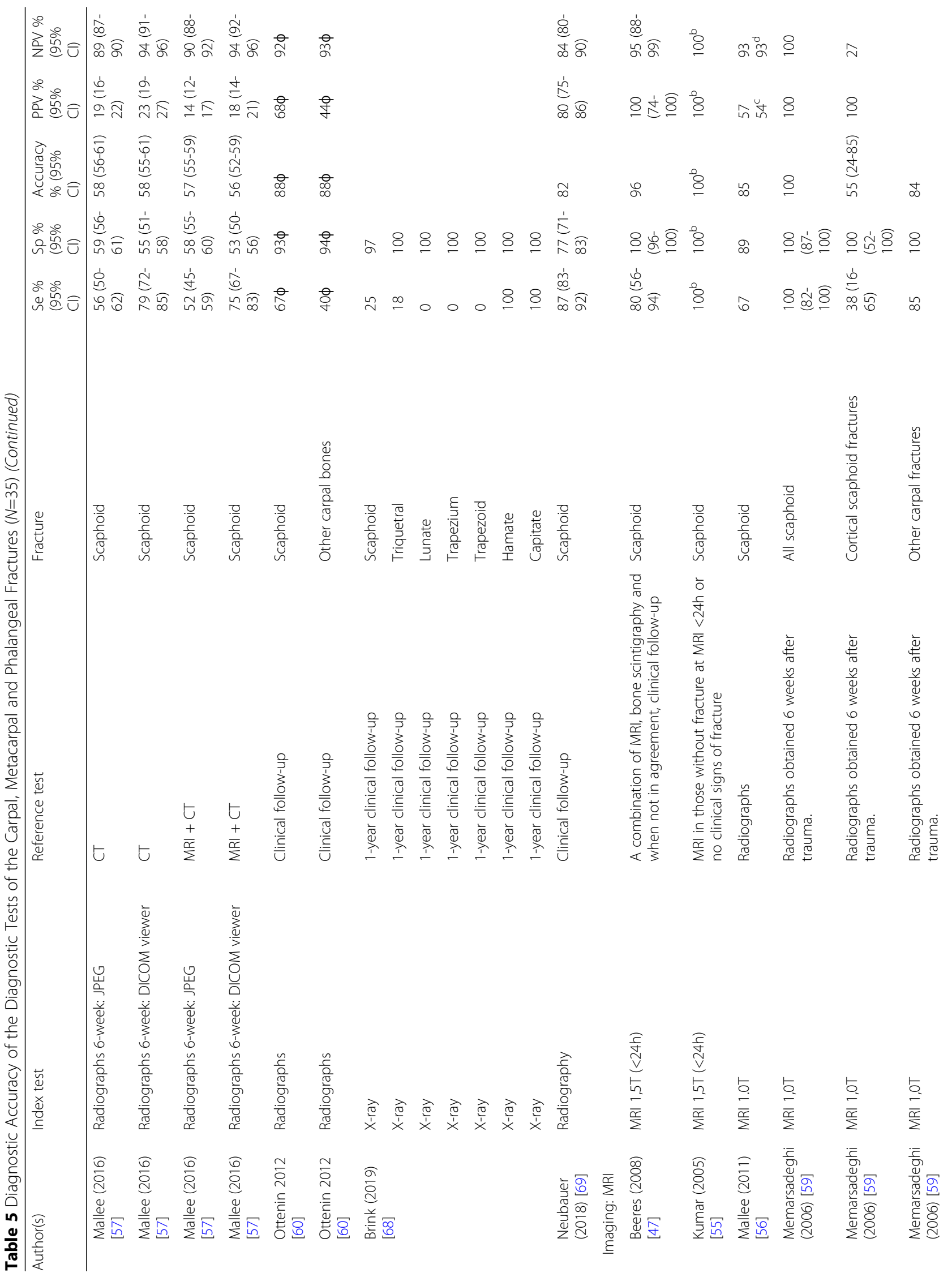




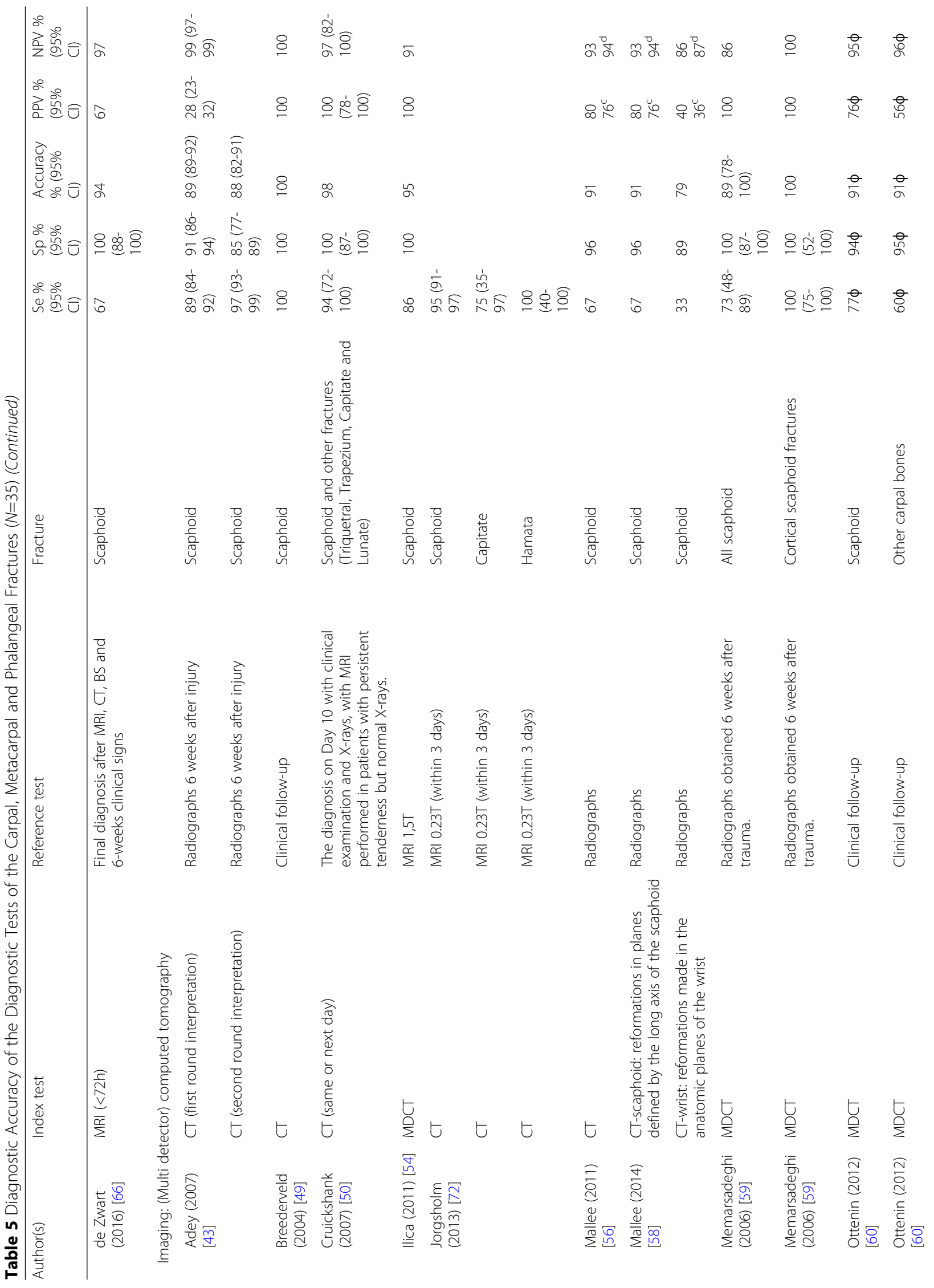




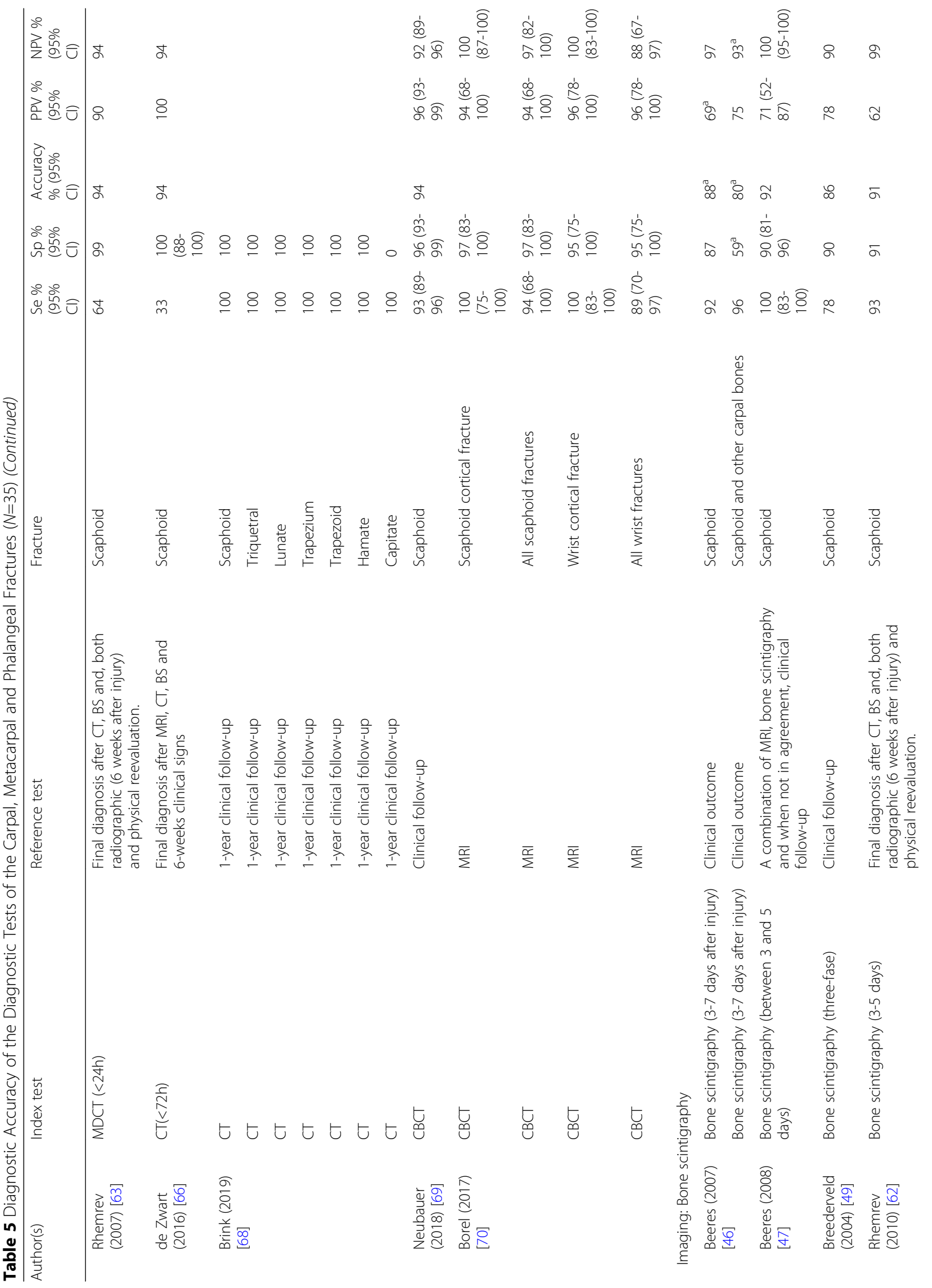




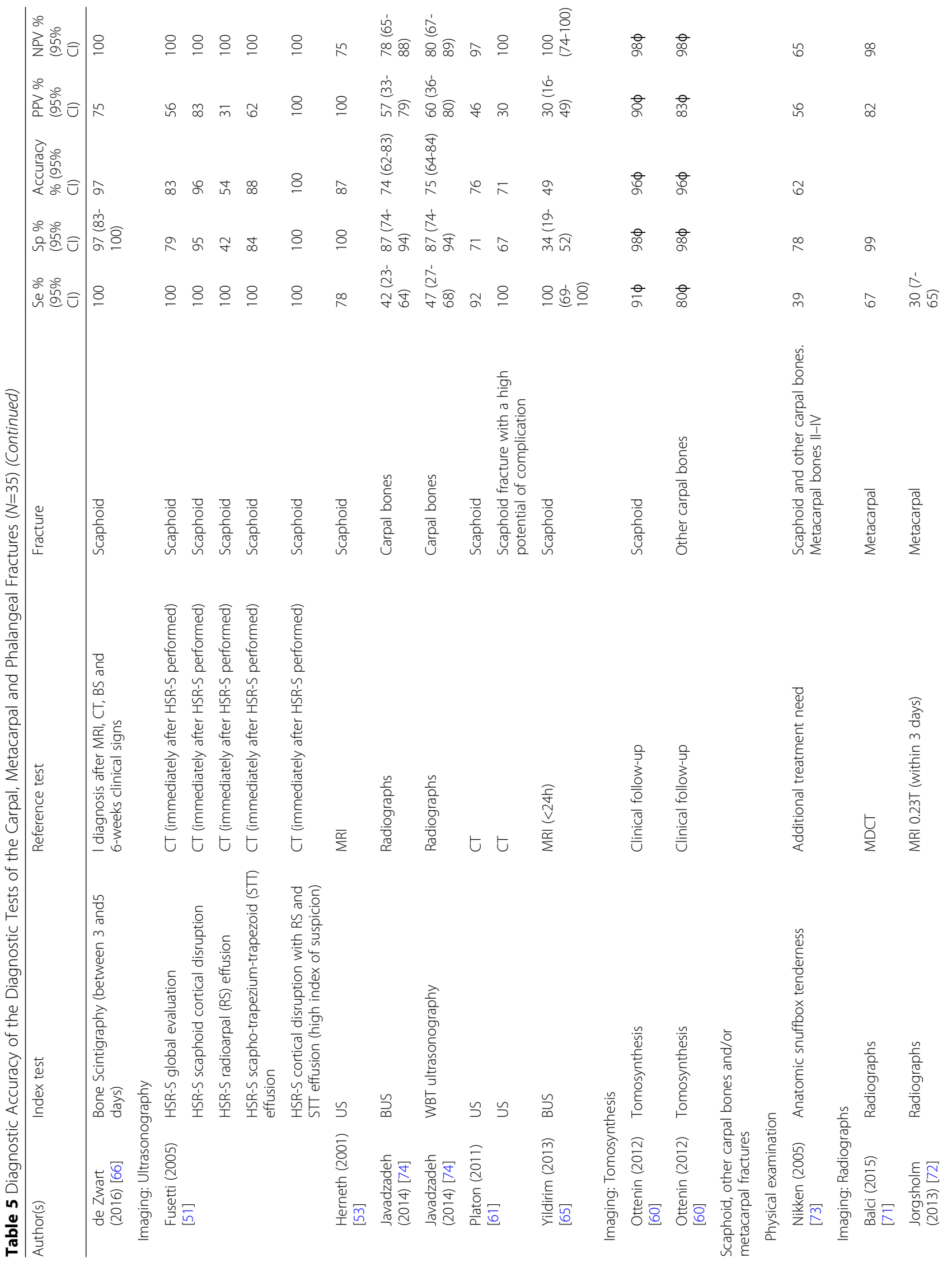




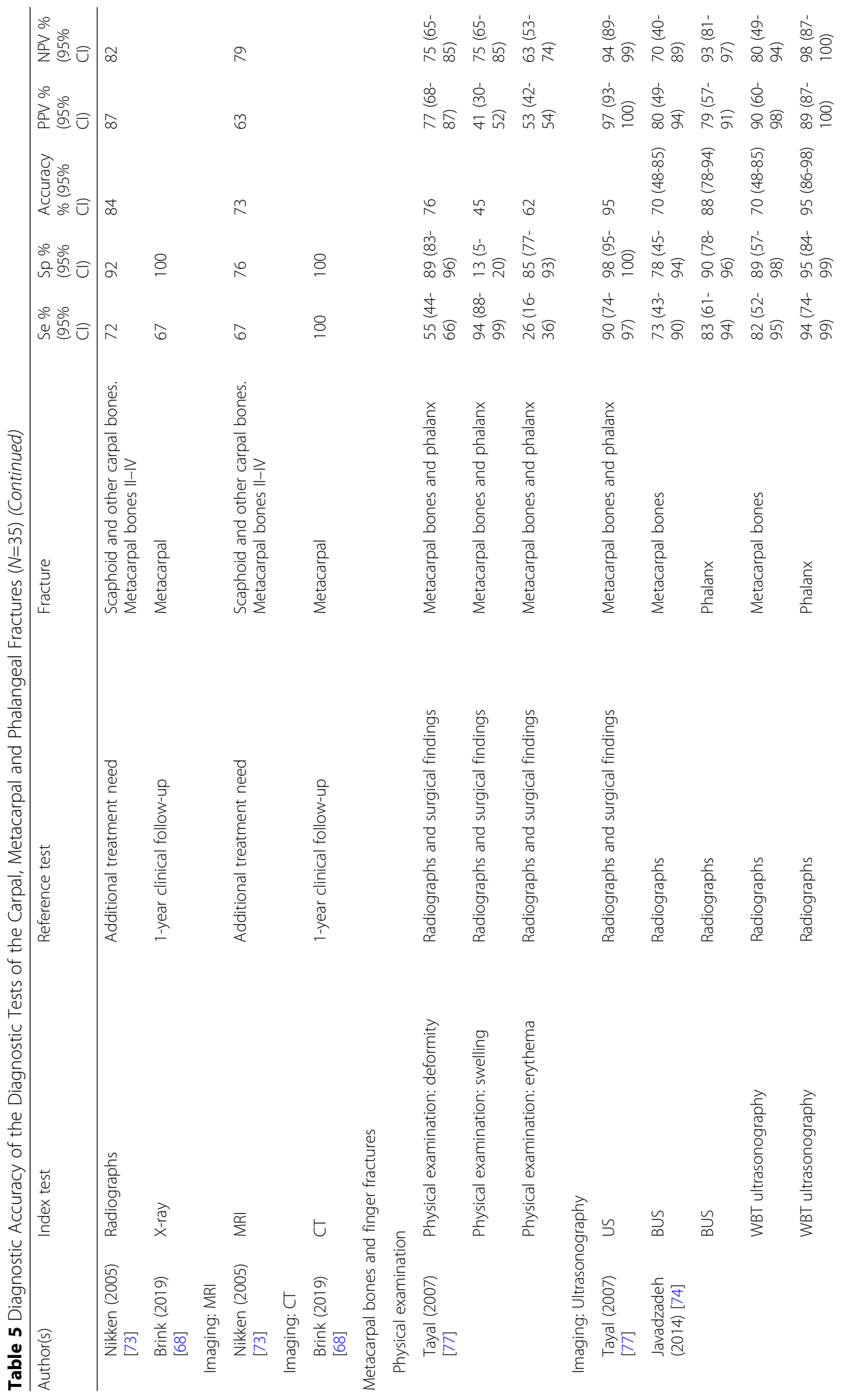




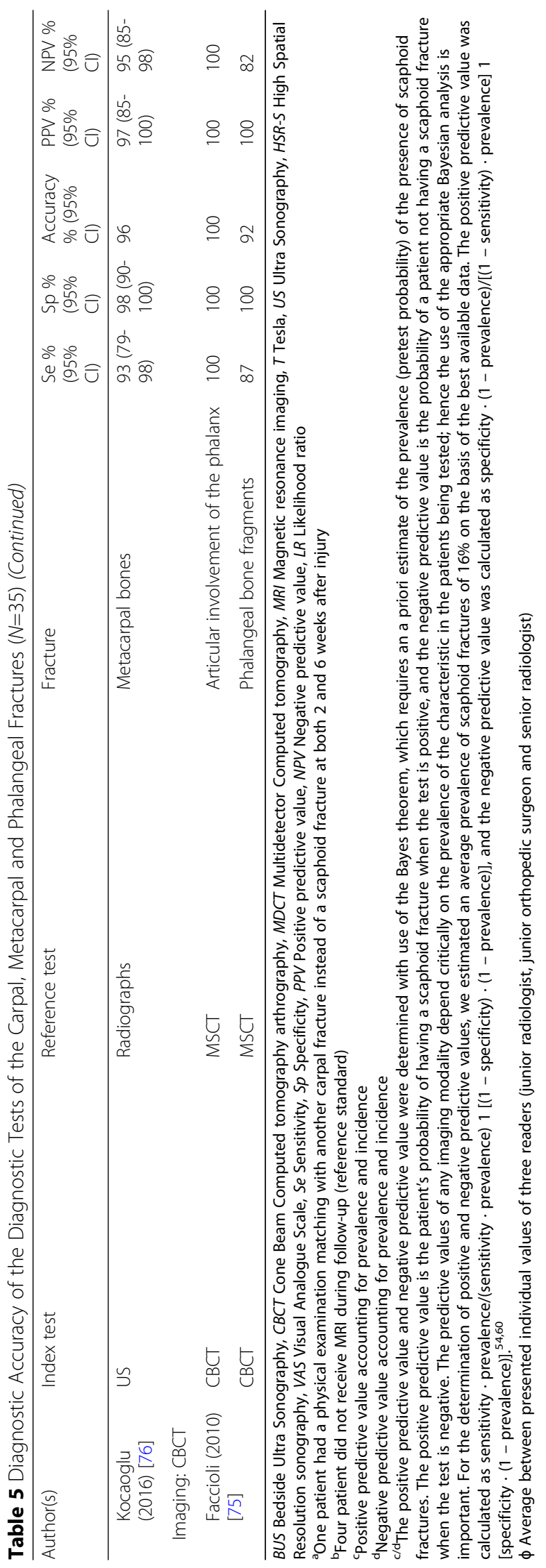


in six studies [71, 73-77]. Physical examination [77] for diagnosing phalangeal and metacarpal fractures showed Se, Sp, accuracy, PPV and NPV ranging from 26 to 55\%, $13-89 \%, 45-76 \%, 41-77 \%$ and $63-75 \%$, respectively. Imaging for metacarpal and finger fractures showed Se, Sp, accuracy, PPV and NPV ranging from 73 to $100 \%$, 78 $100 \%, 70-100 \%, 79-100 \%$ and $70-100 \%$, respectively. The reported diagnostic accuracy measures of phalangeal and metacarpal fractures were characterized by markedly heterogeneous results among the eligible studies.

\section{Combined diagnostic accuracy of the studies with no limitations and no incorporation Bias}

Table 6 shows combined diagnostic accuracy measures of the studies that had no limitations and no incorporation bias. A wide range of results were found for the specificity, accuracy and NPV of MRI, US, CT and BS. The sensitivity of BS and US showed similar, acceptable results. US and MRI are imaging tools that have similar PPV, but with large confidence intervals.

\section{Discussion}

In previous reviews, no studies were identified on the diagnostic accuracy of history taking for phalangeal, metacarpal or carpal fractures. In the current systematic review, only two such studies were identified. This update included one extra study on physical examinations for diagnosing scaphoid fractures in hospital care, which was not included in previous reviews [48]. Based on these results and those presented in the previous reviews, physical examination is of moderate use for diagnosing a scaphoid fracture. Physicians should be aware that tenderness in the anatomical snuff box (ASB), tenderness over the scaphoid tubercle and pain on longitudinal compression of the thumb have limited added value in a diagnostic process for a scaphoid fracture.

The present systematic review identified eight supplementary imaging studies $[58,61,65,66,68-70,74]$, subdivided into MRI [66], CT [58, 66, 68-70], BS [66] and US $[61,65,74]$. The overall conclusion is that imaging tests were found to be moderately accurate for a definitive diagnosis. However, the standard diagnostic workup for wrist complaints suspected of being a fracture should also include detailed patient history taking, a conscientious physical examination and, only if needed, imaging [23]. Diagnostic studies focusing on history taking and physical examination of patients with suspected phalangeal, metacarpal and carpal fractures are therefore desired.

Compared with previous reviews, the current systematic review attempted to distinguish between studies based on their setting. Remarkably, no studies examined the diagnostic accuracy of any diagnostic test for phalangeal, metacarpal and carpal fractures in a non-institutionalized general practitioner care setting. It is known that results from hospital care cannot automatically be translated into guidelines for non-institutionalized general practitioner care. For that reason, it is not possible to advise general practitioners properly on the diagnosis of carpal, metacarpal and phalangeal fractures based on the currently available literature. Given the burden of finger, hand and wrist fractures on non-institutionalized care and the importance of proper diagnoses, diagnostic studies focusing on phalangeal, metacarpal and carpal fractures in non-institutionalized general practitioner care are urgently needed [2].

Table 6 Combined Diagnostic Accuracy of the Studies with no Limitations on QUADAS-2 and No Incorporation Bias ( $N=7$ )

\begin{tabular}{|c|c|c|c|c|c|c|c|}
\hline Author(s) & Diagnostic test & Scaphoid fracture & Se $\%$ & $\mathrm{Sp} \%$ & Accuracy \% & PPV \% & NPV \% \\
\hline Gabler (2001) [52] & $\begin{array}{l}\text { Repeated clinical and } \\
\text { radiological examinations }\end{array}$ & Scaphoid & $82-100$ & 100 & 100 & 100 & 100 \\
\hline Mallee (2016) [57] & Radiographs ${ }^{b}$ & Scaphoid & $42-79$ & $53-59$ & $53-58$ & $14-26$ & 79-94 \\
\hline $\begin{array}{l}\text { Fusetti (2005) [51] and } \\
\text { Platon (2011) [61] }\end{array}$ & Ultrasonography & Scaphoid & $92-100$ & $42-100$ & 54-100 & $30-100$ & $97-100$ \\
\hline Mallee (2011) [56] & MRI & Scaphoid & 67 & 8 & 85 & 57 & 93 \\
\hline $\begin{array}{l}\text { Mallee (2011) [56] and } \\
\text { Mallee (2014 [58] }\end{array}$ & $(\mathrm{MD}) \mathrm{CT}^{\mathrm{C}}$ & Scaphoid & $33-67$ & $89-96$ & 79-91 & $40-80$ & $86-93$ \\
\hline Borel (2017) [70] & $\mathrm{CBCT}$ & Scaphoid & 94 & 97 & & 94 & 97 \\
\hline Author & Diagnostic test & Other carpal fracture & Sensitivity \% & Specificity \% & Accuracy \% & PPV \% & NPV \% \\
\hline Mallee (2014) [58] & $\begin{array}{l}\text { Repeated clinical and } \\
\text { radiological examinations }\end{array}$ & Other carpal bones & $75-100$ & & & & \\
\hline
\end{tabular}

${ }^{a}$ Repeated clinical and radiological examinations after 10 and 38 days

${ }^{b}$ Radiographs after 6 weeks evaluated with JPEG or DICOM files

${ }^{c} \mathrm{CT}$-scaphoid: reformations in planes defined by the long axis of the scaphoid versus CT-wrist: reformations made in the anatomic planes of the wrist 


\section{Methodological quality assessment}

The methodological quality of the eligible studies included in this update was limited, which might affect the estimates of diagnostic accuracy. Many of the included studies had methodological flaws and lacked the necessary details to replicate the studies. There was considerable underreporting of important domains in most of the included studies. The studies in this and previous systematic reviews also had the inherent risk of publication bias. As the mechanisms of publication bias are not yet well understood for diagnostic accuracy studies, there are currently no assessment tools available to investigate this risk other than graphical interpretation. Furthermore, several studies demonstrate incorporation bias, with the risk of overestimation of the diagnostic accuracy [78].

\section{Diagnostic accuracy of the diagnostic tests for phalangeal and metacarpal fractures}

The identified studies evaluated a variety of metacarpal and phalangeal pathologies. US may be an option for detecting metacarpal fractures and prevent unnecessary Xray imaging examinations in patients presenting to the Emergency Department (ED) with hand trauma. Some advantages of US have increased its utilization in emergency departments; these include a short procedure time, a non-invasive and nonionizing radiation involving nature, availability for use in nonhospital settings or bedside settings, repeatability, and a higher safety in children and pregnant patients [79].

None of the previous reviews included studies showing evidence on the diagnostic accuracy for diagnosing metacarpal and phalangeal fractures. Therefore, this is the first study to systematically summarize the diagnostic accuracy of diagnostic tests for phalangeal and metacarpal fractures. This study concludes that physical examination was of limited use for diagnosing phalangeal and metacarpal fractures.

\section{Diagnostic accuracy of history taking and physical examination of carpal fractures}

History taking and physical examination are important tools in a diagnostic process of diagnosing patients with wrist pain [23]. Although common practice in hospital care, only two studies were found on the diagnostic accuracy of history taking for carpal fractures in the previous reviews and current review.

Previous reviews reported that tenderness in the anatomical snuff box demonstrated an Se and Sp for scaphoid fractures ranging from 87 to $100 \%$ and $3-98 \%$, respectively [32, 34]. Tenderness over the scaphoid tubercle (ST) demonstrated a Se and Sp ranging from 82 to $100 \%$ and $17-57 \%$, respectively [32, 34]. The Longitudinal Thumb Compression test (LTC) demonstrated a
Se and Sp ranging from 48 to $100 \%$ and $22-97 \%$, respectively [32, 34].

The current systematic update included three extra studies on physical examinations for diagnosing scaphoid fractures in hospital care $[48,52,53]$. Based on these results and those presented in the previous reviews, combining provocative tests improved the accuracy of the post-test fracture probability, and physical examination alone was not sufficient to rule in or rule out scaphoid fracture, which may lead to unnecessary outpatient reviews and/or overtreatment. If a patient with wrist pain and normal $\mathrm{X}$-rays has a combination of tenderness in the anatomical snuff box, tenderness over the scaphoid tubercle and longitudinal compression (LC) tenderness towards the scaphoid, supplementary imaging is still recommended. At present, in a patient with a strong suspicion of a scaphoid fracture based on history taking and physical examination despite no deviation on imaging, the wrist will be temporarily immobilized until repeated evaluation of the physical examination and imaging has taken place later [80].

\section{Diagnostic accuracy of imaging of carpal fractures}

In this and previous systematic reviews, the reported diagnostic accuracy measures for imaging modalities were characterized by markedly heterogeneous results among the eligible studies. Plain radiography remained the commonest modality for diagnosing carpal fractures [81-83]. Its advantages include its wide availability, easy accessibility and low costs. Most studies describe diagnostic tests of scaphoid fractures and only a few studies concern other carpal fractures. At present, there is still insufficient scientific evidence regarding the ideal imaging technique for scaphoid fractures [23]. Repeated radiographs seems to have limited value for evaluating suspected scaphoid fractures. The irregular contour, the three-dimensional location in the wrist of the scaphoid and the overlap of the carpal bones render interpretation of scaphoid radiographs difficult, especially in the absence of fracture dislocation [81-83].

The best diagnostic modality for confirmation of the diagnosis of a carpal fracture that is not visible on the initial radiograph is still the subject of debate. As found in previous reviews (Table 1), MRI, CT and BS have been shown to have better diagnostic performance than isolated repeated scaphoid radiographs. Previous reviews by Yin et al. concluded that BS and MRI have equally high pooled sensitivity and high diagnostic value for excluding scaphoid fracture, when the lack of a reference standard is acknowledged [35, 36]. However, MRI is more specific and better for confirming scaphoid fractures when compared to BS. According to the Cochrane review of Mallee et al., statistically BS is the best diagnostic modality for establishing a definitive diagnosis in 
clinically suspected fractures when radiographs appear normal, but the number of overtreated patients is substantially lower with CT and MRI [39]. Moreover, physicians must keep in mind that BS is more invasive than the other modalities. Previous reviews by Kwee et al. and Ali et al. concluded that US can diagnose occult scaphoid fracture with a fairly high degree of accuracy and Kwee et al. stated that US may be used when CT and MRI are not readily available $[37,38]$. Nonetheless, one needs to keep in mind that, although scaphoid fractures are the most frequently injured carpal bones, the consequences of fractures of other carpal bones should not be underestimated. All previously available systematic reviews only examined diagnostic tests for scaphoid fractures [32-39], while in practice it is often not quite clear during the diagnostic process which hand or wrist anatomical structure or tissue (soft tissue or bone) is affected.

\section{Conclusion}

As no studies in non-institutionalized general practitioner care were identified, general practitioners who examine patients with a suspected hand or wrist fracture have limited instruments for providing adequate diagnostics. A general practitioner could decide to refer such patients to a hospital for specialized care, but one could question what assessments a specialist can use to come to an accurate diagnosis. In hospital care, two studies of the diagnostic accuracy of history taking for phalangeal, metacarpal and carpal fractures were found and physical examination was of moderate use for diagnosing a scaphoid fracture and of limited use for diagnosing phalangeal, metacarpal and remaining carpal fractures. Based on the best evidence synthesis, imaging tests (conventional radiograph, MRI, CT and BS) were only found to be moderately accurate for definitive diagnosis in hospital care.

\section{Abbreviations}

ASB: Anatomic snuff-box; BS: Bone scintigraphy; BUS: Bedside ultra sonography; CBCT:: Cone beam computer tomography; CT: Computed tomography; HR: High risk; HSR-S: High spatial resolution-sonography; LR: Likelihood ratio; LTC: Longitudinal (thumb) compression test; MDCT: Multi detector computed tomography; MRI: Magnetic resonance imaging; MSCT: Multi-slice computer tomography; NPV: Negative predictive value; PPV: Positive predictive value; QUADAS: Quality Assessment of diagnostic accuracy studies; ROM: Range of motion; Se: Sensitivity; Sp: Specificity; STT: Scaphoid tubercle tenderness; T: Tesla; UR: Unclear Risk; US: Ultra sonography; VAS: Visual analogue scale; WBT: Water bath technique

\section{Acknowledgements}

The authors thank Wichor Bramer (Biomedical information specialist of Erasmus MC University Medical Center Rotterdam, Medical Library) for help with the electronic search strategies and Yassine Aaboubout (MSc) for helping with study selection and extracting the data.

\section{Authors' contributions}

PK, NM, SB, GK and JR all contributed to the design of the study. PK and JR were responsible for article selection and analysed the data. All authors contributed to writing and revision of the manuscript. All authors have given approval of the submitted version of the manuscript and agree to be accountable for all aspects of the work.

\section{Funding}

No funding.

\section{Availability of data and materials}

The datasets used and/or analysed during the current study are available from the corresponding author on reasonable request.

Ethics approval and consent to participate

Not applicable.

\section{Consent for publication}

Not applicable.

\section{Competing interests}

The authors declare that they have no competing interests.

\section{Author details}

'Department of General Practice, Erasmus MC University Medical Center Rotterdam, Room NA1911 PO Box 2040, 3000, CA, Rotterdam, the Netherlands. ${ }^{2}$ Department of Orthopaedic Surgery, Reinier de Graaf Groep, Reinier de Graafweg 5-11, 2625, AD, Delft, the Netherlands. ${ }^{3}$ Department of Orthopaedics, Erasmus MC University Medical Center Rotterdam, Room NA1920 PO Box 2040, 3000, CA, Rotterdam, the Netherlands. ${ }^{4}$ Department of General Practice, Erasmus MC University Medical Center Rotterdam, Room NA1920 PO Box 2040, 3000, CA, Rotterdam, the Netherlands.

Received: 3 September 2019 Accepted: 4 December 2019

Published online: 07 January 2020

\section{References}

1. Owen RA, Melton $L \mathrm{III}$, Johnson KA, Ilstrup DM, Riggs BL. Incidence of Colles fracture in a north American community. Am J Public Health. 1982; 72:605-7.

2. Larsen CF, Mulder S, Johansen AM, Stam C. The epidemiology of hand injuries in The Netherlands and Denmark. Eur J Epidemiol. 2004;19(4):323-7.

3. McCullough NP, Smith FW, Cooper JG. Early MRI in the management of the clinical scaphoid fracture. Eur J Emerg Med. 2011;18(3):133-6.

4. van der Molen AB, Groothoff JW, Visser GJ, Robinson PH, Eisma WH. Time off work due to scaphoid fractures and other carpal injuries in the Netherlands in the period 1990 to 1993. J Hand Surg Br. 1999;24(2):193-8.

5. Hey HWD, Chong AKS, Murphy D. Prevalence of carpal fracture in Singapore. J Hand Surg Am. 2011;36(2):278-83.

6. Van Onselen EB, Karim RB, Hage JJ, Ritt MJ. Prevalence and distribution of hand fractures. J Hand Surg Br. 2003;28(5):491-5.

7. Cooney WP III. Scaphoid fractures: current treatments and techniques. Instr Course Lect. 2003;52:197-208.

8. Krasin E, Goldwirth M, Gold A, Goodwin DR. Review of the current methods in the diagnosis and treatment of scaphoid fractures. Postgrad Med J. 2001; 77:235-7.

9. Frazier WH, Miller M, Fox RS, Brand D, Finseth F. Hand injuries: incidence and epidemiology in an emergency service. JACEP. 1978;7:265-8.

10. Aitken S, Court-Brown CM. The epidemiology of sports-related fractures of the hand. Injury. 2008;39:1377-83.

11. Van der Linden MW, Westert GP, de Bakker DH, Schellevis FG. Tweede Nationale Studie naar ziekten en verrichtingen in de huisartspraktijk. NIVEL/ RIVM: Klachten en aandoeningen in de bevolking en in de huisartspraktijk. Utrecht/Bilthoven; 2004.

12. Roolker W, Maas M, Broekhuizen AH. Diagnosis and treatment of scaphoid fractures, can non union be prevented? Arch Orthop Trauma Surg. 1999; 119:428-31.

13. Schaub TA, Chung KC. Systems of provision and delivery of hand care, and its impact on the community. Injury. 2006;37(11):1066-70.

14. de Putter CE, van Beeck EF, Polinder S, Panneman MJ, Burdorf A, Hovius SE, Selles RW. Healthcare costs and productivity costs of hand and wrist injuries by external cause: a population-based study in working-age adults in the period 2008-2012. Injury. 2016;47(7):1478-82. 
15. Greene WB. Essentials of musculoskeletal care. Rosemont, IL: American Academy of Orthopaedic Surgeons, 2001.

16. Langhoff $\mathrm{O}$, Andersen JL. Consequences of late immobilization of scaphoid fractures. J Hand Surg Br. 1988;13:77-9.

17. Eddeland A, Eiken O, Hellgren E, Ohlsson NM. Fractures of the scaphoid. Scand J Plast Reconstr Surg. 1975;9:234-9.

18. Taleisnik J. Clinical and technologic evaluation of ulnar wrist pain. J Hand Surg [Am]. 1988;13:801-2.

19. Steenvoorde P, Jacobi C, van der Lecq A, van Doorn L, Kievit J, Oskam J. Development of a clinical decision tool for suspected scaphoid fractures. Acta Orthop Belg. 2006;72(4):404-10.

20. Phillips TG, Reibach AM, Slomiany WP. Diagnosis and management of scaphoid fractures. Am Fam Physician. 2004;70:879-84.

21. Freeland P. Scaphoid tubercle tenderness: a better indicator of scaphoid fractures? Arch Emerg Med. 1989;6:46-50.

22. Grover R. Clinical assessment of scaphoid injuries and the detection of fractures. J Hand Surg Br. 1996;21:341-3.

23. Groves AM, Kayani I, Syed R, Hutton BF, Bearcroft PP, Dixon AK, Ell PJ. An international survey of hospital practice in the imaging of acute scaphoid trauma. AJR Am J Roentgenol. 2006;187(6):1453-6.

24. Hobby JL, Tom BD, Bearcroft PW, Dixon AK. Magnetic resonance imaging of the wrist: diagnostic performance statistics. Clin Radiol. 2001;56(1):50-7.

25. Tiel-van Buul MM, van Beek EJ, Borm JJ, Gubler FM, Broekhuizen AH, van Royen EA. The value of radiographs and bone scintigraphy in suspected scaphoid fracture. A statistical analysis. J Hand Surg Br. 1993;18:403-6.

26. Hunter JC, Escobedo EM, Wilson AJ, Hanel DP, Zink-Brody GC, Mann FA. MR imaging of clinically suspected scaphoid fractures. AJR Am J Roentgenol. 1997;168:1287-93.

27. Furunes H, Vandvik PO. Cast immobilisation for suspected scaphoid fractures. Tidsskr Nor Laegeforen. 2009;129:177-9.

28. Cheung GC, Lever CJ, Morris AD. X-ray diagnosis of acute scaphoid. J Hand Surg Br. 2006;31:104-9.

29. Lozano-Calderon S, Blazar P, Zurakowski D, Lee SG, Ring D. Diagnosis of scaphoid fracture displacement with radiography and computed tomography. J Bone Joint Surg Am. 2006;88:2695-703.

30. Steel N, Abdelhamid A, Stokes T, Edwards H, Fleetcroft R, Howe A, Qureshi $N$. A review of clinical practice guidelines found that they were often based on evidence of uncertain relevance to primary care patients. J Clin Epidemiol. 2014;67(11):1251-7.

31. Ring D, Lozano-Calderon S. Imaging for suspected scaphoid fracture. J Hand Surg Am. 2008;33(6):954-7.

32. Carpenter CR, Pines JM, Schuur JD, Muir M, Calfee RP, Raja AS. Adult scaphoid fracture. Acad Emerg Med. 2014;21(2):101-21.

33. Burrows B, Moreira P, Murphy C, Sadi J, Walton DM. Scaphoid fractures: a higher order analysis of clinical tests and application of clinical reasoning strategies. Man Ther. 2014;19(5):372-8.

34. Mallee WH, Henny EP, van Dijk CN, Kamminga SP, van Enst WA, Kloen P. Clinical diagnostic evaluation for scaphoid fractures: a systematic review and meta-analysis. J Hand Surg Am. 2014;39(9):1683-91.

35. Yin ZG, Zhang JB, Kan SL, Wang XG. Diagnostic accuracy of imaging modalities for suspected scaphoid fractures: meta-analysis combined with latent class analysis. J Bone Joint Surg Br. 2012;94(8):1077-85.

36. Yin ZG, Zhang JB, Kan SL, Wang XG. Diagnosing suspected scaphoid fractures: a systematic review and meta-analysis. Clin Orthop Relat Res. 2010;468(3):723-34.

37. Kwee RM, Kwee TC. Ultrasound for diagnosing radiographically occult scaphoid fracture. Skelet Radiol. 2018;47(9):1205-12.

38. Ali M, Ali M, Mohamed A, Mannan S, Fallahi F. The role of ultrasonography in the diagnosis of occult scaphoid fractures. J Ultrason. 2018;18(75):325-31.

39. Mallee WH, Wang J, Poolman RW, Kloen P, Maas M, de Vet HCW, Doornberg $\mathrm{JN}$. Computed tomography versus magnetic resonance imaging versus bone scintigraphy for clinically suspected scaphoid fractures in patients with negative plain radiographs. Cochrane Database of Systematic Reviews 2015, Issue 6. Art. No.: CD010023.

40. Moher D, Liberati A, Tetzlaff J, Altman DG; PRISMA group Preferred reporting items for systematic reviews and meta-analyses: the PRISMA statement BMJ 2009; 339: b2535.

41. Whiting PF, Rutjes AW, Westwood ME, Mallett S, Deeks JJ, Reitsma JB, Leeflang MM, Sterne JA, Bossuyt PM. QUADAS-2: a revised tool for the quality assessment of diagnostic accuracy studies. QUADAS-2 group. Ann Intern Med. 2011;155(8):529-36.
42. Devillé WL, Buntinx F, Bouter LM, Montori VM, de Vet HC, van der Windt DA, Bezemer PD. Conducting systematic reviews of diagnostic studies: didactic guidelines. BMC Med Res Methodol. 2002;2:9.

43. Adey L, Souer JS, Lozano-Calderon S, Palmer W, Lee SG, Ring D. Computed tomography of suspected scaphoid fractures. J Hand Surg Am. 2007;32(1): 61-6.

44. Annamalai G, Raby N. Scaphoid and pronator fat stripes are unreliable soft tissue signs in the detection of radiographically occult fractures. Clin Radiol. 2003;58(10):798-800

45. Behzadi C, Karul M, Henes FO, Laqmani A, Catala-Lehnen P, Lehmann W, Nagel HD, Adam G, Regier M. Comparison of conventional radiography and MDCT in suspected scaphoid fractures. World J Radiol. 2015;7(1):22-7.

46. Beeres FJ, Hogervorst M, Rhemrev SJ, den Hollander P, Jukema GN. A prospective comparison for suspected scaphoid fractures: bone scintigraphy versus clinical outcome. Injury. 2007;38(7):769-74.

47. Beeres FJ, Rhemrev SJ, den Hollander P, Kingma LM, Meylaerts SA, le Cessie S, Bartlema KA, Hamming JF, Hogervorst M. Early magnetic resonance imaging compared with bone scintigraphy in suspected scaphoid fractures. Jone Joint Surg Br. 2008;90(9):1205-9.

48. Bergh TH, Lindau T, Soldal LA, Bernardshaw SV, Behzadi M, Steen K, Brudvik C. Clinical scaphoid score (CSS) to identify scaphoid fracture with MRI in patients with normal X-ray after a wrist trauma. Emerg Med J. 2014;31(8):659-64.

49. Breederveld RS, Tuinebreijer WE. Investigation of computed tomographic scan concurrent criterion validity in doubtful scaphoid fracture of the wrist. J Trauma. 2004;57(4):851-4.

50. Cruickshank J, Meakin A, Breadmore R, Mitchell D, Pincus S, Hughes T, Bently B, Harris M, Vo A. Early computerized tomography accurately determines the presence or absence of scaphoid and other fractures. Emerg Med Australas. 2007 Jun;19(3):223-228. Erratum in: Emerg Med Australas. 2007;19(4):387.

51. Fusetti C, Poletti PA, Pradel PH, Garavaglia G, Platon A, Della Santa DR, Bianchi S. Diagnosis of occult scaphoid fracture with high-spatial-resolution sonography: a prospective blind study. J Trauma. 2005;59(3):677-81.

52. Gäbler C, Kukla C, Breitenseher MJ, Trattnig S, Vécsei V. Diagnosis of occult scaphoid fractures and other wrist injuries. Are repeated clinical examinations and plain radiographs still state of the art? Langenbeck's Arch Surg. 2001;386(2):150-4.

53. Herneth AM, Siegmeth A, Bader TR, Ba-Ssalamah A, Lechner G, Metz VM, Grabenwoeger F. Scaphoid fractures: evaluation with high-spatial-resolution US initial results. Radiol. 2001;220(1):231-5.

54. Ilica AT, Ozyurek S, Kose O, Durusu M. Diagnostic accuracy of multidetector computed tomography for patients with suspected scaphoid fractures and negative radiographic examinations. Jpn J Radiol. 2011;29(2):98-103.

55. Kumar S, O'Connor A, Despois M, Galloway H. Use of early magnetic resonance imaging in the diagnosis of occult scaphoid fractures: the CAST Study (Canberra Area Scaphoid Trial). N Z Med J. 2005;118(1209):U1296.

56. Mallee W, Doornberg JN, Ring D, van Dijk CN, Maas M, Goslings JC. Comparison of CT and MRI for diagnosis of suspected scaphoid fractures. J Bone Joint Surg Am. 2011;93(1):20-8.

57. Mallee WH, Mellema JJ, Guitton TG, Goslings JC, Ring D. Doornberg JN; science of variation group. 6-week radiographs unsuitable for diagnosis of suspected scaphoid fractures. Arch Orthop Trauma Surg. 2016;136(6):771-8.

58. Mallee WH, Doornberg JN, Ring D, Maas M, Muhl M, van Dijk CN, Goslings JC. Computed tomography for suspected scaphoid fractures: comparison of reformations in the plane of the wrist versus the long axis of the scaphoid. Hand (NY). 2014;9(1):117-21.

59. Memarsadeghi M, Breitenseher MJ, Schaefer-Prokop C, Weber M, Aldrian S, Gäbler C, Prokop M. Occult scaphoid fractures: comparison of multidetector CT and MR imaging-initial experience. Radiol. 2006;240(1):169-76.

60. Ottenin MA, Jacquot A, Grospretre O, Noël A, Lecocq S, Louis M, Blum A. Evaluation of the diagnostic performance of tomosynthesis in fractures of the wrist. AJR Am J Roentgenol. 2012;198(1):180-6.

61. Platon A, Poletti PA, Van Aaken J, Fusetti C, Della Santa D, Beaulieu JY, Becker CD. Occult fractures of the scaphoid: the role of ultrasonography in the emergency department. Skelet Radiol. 2011;40(7):869-75.

62. Rhemrev SJ, Beeres FJ, van Leerdam RH, Hogervorst M, Ring D. Clinical prediction rule for suspected scaphoid fractures: A prospective cohort study. Injury. 2010;41(10):1026-30.

63. Rhemrev SJ, de Zwart AD, Kingma LM, Meylaerts SA, Arndt JW, Schipper IB, Beeres FJ. Early computed tomography compared with bone scintigraphy in suspected scaphoid fractures. Clin Nucl Med. 2010;35(12):931-4. 
64. Steenvoorde P, Jacobi C, van Doorn L, Oskam J. Pilot study evaluating a clinical decision tool on suspected scaphoid fractures. Acta Orthop Belg. 2006;72(4):411-4.

65. Yıldırım A, Unlüer EE, Vandenberk N, Karagöz A. The role of bedside ultrasonography for occult scaphoid fractures in the emergency department. Ulus Travma Acil Cerrahi Derg. 2013;19(3):241-5.

66. de Zwart AD, Beeres FJ, Rhemrev SJ, Bartlema K, Schipper IB. Comparison of $\mathrm{MRI}, \mathrm{CT}$ and bone scintigraphy for suspected scaphoid fractures. Eur J Trauma Emerg Surg. 2016;42(6):725-31.

67. Sharifi MD, Moghaddam HZ, Zakeri H, Ebrahimi M, Saeedian H, Hashemian AM. The accuracy of pain measurement in diagnosis of scaphoid bone fractures in patients with magnetic resonance imaging: report of 175 cases. Med Arch. 2015;69(3):161-4.

68. Brink M, Steenbakkers A, Holla M, de Rooy J, Cornelisse S, Edwards MJ, Prokop M. Single-shot CT after wrist trauma: impact on detection accuracy and treatment of fractures. Skelet Radiol. 2019;48(6):949-57.

69. Neubauer J, Benndorf M, Ehritt-Braun C, Reising K, Yilmaz T, Klein C, Zajonc $\mathrm{H}$, Kotter $\mathrm{E}$, Langer M, Goerke SM. Comparison of the diagnostic accuracy of cone beam computed tomography and radiography for scaphoid fractures. Sci Rep. 2018;8(1):3906.

70. Borel C, Larbi A, Delclaux S, Lapegue F, Chiavassa-Gandois H, Sans N, Faruch-Bilfeld M. Diagnostic value of cone beam computed tomography (CBCT) in occult scaphoid and wrist fractures. Eur J Radiol. 2017:97:59-64.

71. Balci A, Basara I, Çekdemir EY, Tetik F, Aktaş G, Acarer A, Özaksoy D. Wrist fractures: sensitivity of radiography, prevalence, and patterns in MDCT. Emerg Radiol. 2015;22(3):251-6.

72. Jørgsholm P, Thomsen NO, Besjakov J, Abrahamsson SO, Björkman A. The benefit of magnetic resonance imaging for patients with posttraumatic radial wrist tenderness. J Hand Surg Am. 2013;38(1):29-33.

73. Nikken JJ, Oei EH, Ginai AZ, Krestin GP, Verhaar JA, van Vugt AB, Hunink MG. Acute wrist trauma: value of a short dedicated extremity MR imaging examination in prediction of need for treatment. Radiol. 2005;234(1):116-24.

74. Javadzadeh HR, Davoudi A, Davoudi F, Ghane MR, Khajepoor H, Goodarzi H, Faraji M, Mahmoudi S, Shariat SS, Emami MK. Diagnostic value of "bedside ultrasonography" and the "water bath technique" in distal forearm, wrist, and hand bone fractures. Emerg Radiol. 2014;21(1):1-4.

75. Faccioli N, Foti G, Barillari M, Atzei A, Mucelli RP. Finger fractures imaging: accuracy of cone-beam computed tomography and multislice computed tomography. Skelet Radiol. 2010;39(11):1087-95.

76. Kocaoğlu S, Özhasenekler A, İçme F, Pamukçu Günaydın G, Şener A, Gökhan Ş. The role of ultrasonography in the diagnosis of metacarpal fractures. Am J Emerg Med. 2016;34(9):1868-71.

77. Tayal VS, Antoniazzi J, Pariyadath M, Norton HJ. Prospective use of ultrasound imaging to detect bony hand injuries in adults. J Ultrasound Med. 2007;26(9):1143-8.

78. Worster A, Carpenter C. Incorporation bias in studies of diagnostic tests: how to avoid being biased about bias. CJEM. 2008;10(2):174-5.

79. William DM, Kurtz BK, Hertzberg BS. In: Yımaz C, editor. Bilinmesi GerekenlerUltrason. Çev. Ed. 2. Baskı. İzmir: Güven Bilimsel; 2008:3-4.

80. Dias J, Kantharuban S. Treatment of scaphoid fractures: European approaches. Hand Clin. 2017;33(3):501-9.

81. Low G, Raby N. Can follow-up radiography for acute scaphoid fracture still be considered a valid investigation? Clin Radiol. 2005;60:1106-10.

82. Munk B, Frokjaer J, Larsen CF, Johannsen HG, Rasmussen LL, Edal A, Rasmussen LD. Diagnosis of scaphoid fractures. A prospective multicenter study of 1,052 patients with 160 fractures. Acta OrthopScand. 1995;66:359-60.

83. Tiel-van Buul MM, van Beek EJ, Broekhuizen AH, Nooitgedacht EA, Davids $\mathrm{PH}$, Bakker AJ. Diagnosing scaphoid fractures: radiographs cannot be used as a gold standard! Injury. 1992;23:77-9.

\section{Publisher's Note}

Springer Nature remains neutral with regard to jurisdictional claims in published maps and institutional affiliations.

Ready to submit your research? Choose BMC and benefit from:

- fast, convenient online submission

- thorough peer review by experienced researchers in your field

- rapid publication on acceptance

- support for research data, including large and complex data types

- gold Open Access which fosters wider collaboration and increased citations

- maximum visibility for your research: over $100 \mathrm{M}$ website views per year

At BMC, research is always in progress.

Learn more biomedcentral.com/submissions 